\title{
Spin Density Accuracy and Distribution in Azido Cu(II) Complexes: A Source Function Analysis
}

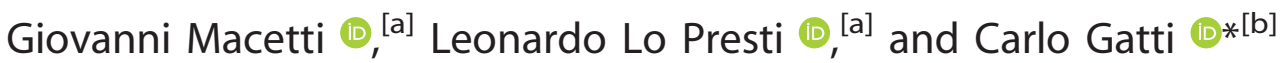

\begin{abstract}
Magnetic properties of open-shell systems depend on their unpaired electron density distribution. Accurate spin density $(S D)$ is difficult to retrieve, both from polarized neutron diffraction (PND) data and from quantum approaches, and its interpretation is not trivial. The Source Function is a useful tool to interpret SD distributions and their accuracy. It is here applied to analyze and compare the theoretical SD in a weakly ferromagnetically coupled end-end azido dicopper complex with that in a strongly-coupled end-on complex. The Source Function enables to highlight the origin of the SD differences between the two dicopper complexes and among adopted
\end{abstract}

computational approaches (CASSCF, DFT, UHF). Further insight is provided by partial Source Function SD reconstructions using given subsets of atoms. DFT methods exaggerate electron sharing between copper and the ligands, causing spin delocalization toward them and overestimating metal-ligand spin polarization, while underestimating CASSCF spin information transmission between atoms. $\operatorname{CAS}(10,10)$ SD is closer to the PND SD than other adopted methods (c) 2018 Wiley Periodicals, Inc.

DOI: $10.1002 / j c c .25150$

\section{Introduction}

Spin density (SD) distribution $s(\boldsymbol{r})$, which is defined as the difference between the $\alpha$ and $\beta$ components of the electron density (ED) $\rho(\boldsymbol{r})$, is certainly one of the most useful quantities to study the spin information transmission in a system. Understanding the magnetic interaction pathways and the mechanisms through which magnetic and non-magnetic centers communicate is an obligatory step to project specific magnetic networks and to tune their properties. ${ }^{[1-3]}$ In the last half-century, the charge density community has focused its attention mainly on the study of the ED topology, testified by large number of key-stone papers and introducing new mathematical topological descriptors able to enhance its chemical interpretability. ${ }^{[4-7]}$ If we can consider the study of the ED as a "mature" (but not saturated) field, this is not the case for the SD. The reasons of this lack of knowledge lie in its intrinsic nature and in the technical difficulties to retrieve accurate enough SDs. Experimentally, the $s(\boldsymbol{r})$ may be derived from polarized neutron diffraction (PND) experiment on a magnetic crystal, which requires a neutron source and a sophisticated setup. ${ }^{[8,9]}$ The very small number of reflections obtainable from PND experiment (only $\sim 10 \%$ of all the reflections in the reciprocal space) and the restricted access to neutron beamtime are the most important factors that hinder the reconstruction of good experimental SD distributions. $^{[3]} \mathrm{A}$ boost in this direction has been recently achieved by Deutsch et al., who introduced for the first time a spin-split $\left(\rho_{\alpha}\right.$ and $\rho_{\beta}$ ) version of the Hansen and Coppens multipolar model ${ }^{[10]}$ for a joined fitting of X-ray and PND data. ${ }^{[3]}$ Through this approach, they demonstrate that experimentally refined $\rho_{\alpha}$ and $\rho_{\beta}$ distributions are good enough to be comparable to the theoretical results, ${ }^{[2,3]}$ although some important warning was recently put forward by us as to the insufficiency of such agreement to also have comparable SDs. ${ }^{[11]}$ Theoretical SDs can be easily calculated using, for example, unrestricted methods, but they are usually far from being reliable. Systematic studies using density functional theory (DFT) exchange-correlation functionals, which are largely used in literature, have demonstrated that they are unable to treat open-shell systems properly, leading to nonaccurate SDs. ${ }^{[12-14]} A b$ initio electron correlation methods are needed to predict more reliable SDs, but they are sometimes too computational demanding and cannot be used for large systems.

In addition to these technical problems, the extraction of chemical information from the SD is not an easy task and it is for sure less trivial than for $\rho(\boldsymbol{r})$, even for very simple molecules such as triplet state water or alkyls radicals. ${ }^{[15,16]}$ For this purpose, in some previous works the concept of Source Function (SF), first introduced in $1998,{ }^{[17]}$ has been extended to the SD distribution. ${ }^{[15,16]}$ The SF descriptor enables one to visualize either the ED or the SD distributions in terms of a cause-effect relationship, where $\rho(\boldsymbol{r})$ or $s(\boldsymbol{r})$ at any point can be envisaged as due to an associated local source operating at any other point $\boldsymbol{r}^{\prime}$ in the space. ${ }^{[18]}$ When the molecule is partitioned according to the Quantum Theory of Atoms In Molecules (QTAIM), ${ }^{[19]}$ the SF provides insight on the ability of an atom or a group of atoms to contribute to the reconstruction of $\rho(\boldsymbol{r})$ or $s(\boldsymbol{r})$ in any specific reference point (RP). In the case of $s(\boldsymbol{r})$ reconstruction, the SF tool highlights the magnetic molecular pattern for the selected molecular or crystal region.

[a] G. Macetti, L. Lo Presti

Department of Chemistry, Università degli Studi di Milano, via Golgi 19, Milano 1-20133, Italy

[b] C. Gatti

Istituto di Scienze e Tecnologie Molecolari (ISTM), Consiglio Nazionale delle Ricerche (CNR), via Golgi 19, Milano I-20133, Italy

E-mail: c.gatti@istm.cnr.it

Contract grant sponsor: Danmarks Grundforskningsfond; Contract grant number: DNRF93

(c) 2018 Wiley Periodicals, Inc. 
In our latest work, we have tried to push up the limits of the SD interpretation through the SF tool by analyzing the magnetic molecular patterns of more complicated cases, namely two azido dicopper complexes. ${ }^{[1]}$ The SF descriptor applied to the first of this couple of compounds, a symmetric end-on (EO) azide, ${ }^{[20]}$ was able to neatly identify the similarities and the differences between the SD distributions obtained with different theoretical methods. It has been shown that the DFT functional exaggerates electron sharing between copper and the ligands, causing spin delocalization toward them and overestimating spin polarization mechanisms, while underestimating spin information transmission relative to the complete active space self-consistent field (CASSCF or CAS) method. Due to the large complexity of the SD distribution, the choice of proper RPs for the SF analysis and the interpretation of their results are not as straightforward as are for the study of the ED. To overcome this problem, two-dimensional (2D) SF reconstruction has been recently proposed. ${ }^{[11]}$ Two- and threedimensional SF maps allow one to see how a subset of atoms reconstructs the SD in a plane or in a portion of space, differentiating informative regions from the non-informative ones and simplifying this choice. To improve the chemical comprehensibility and to provide new insights, spin density SF can be decomposed in two complementary contributions: a magnetic term, which takes into account the contribution from the singly occupied orbitals, and a relaxation term due to the remaining orbitals. ${ }^{[11,15]}$ The high sensitivity of the SF descriptor allows it to be also a valid tool to bring to light small differences and to discuss the accuracy of SD distributions obtained from different calculation methodologies or to compare experiment and theory outcomes. ${ }^{[11]}$

In this article, we want to complete the study of the theoretical $\mathrm{SD}$ in azido $\mathrm{Cu}^{\text {II }}$ complexes analyzing the end-end (EE) system. ${ }^{\text {[21] }}$ Two are the main purposes of this work. First of all, we want to perform a comparison between this EE complex and the EO studied in the previous case. ${ }^{[11]}$ In both systems, the Cu centers are ferromagnetically coupled, but with quite different coupling constant $J$, large for the EO and almost negligible for the EE system. ${ }^{[20,21]}$ Second, we want to largely deepen our tests on the accuracy of the SDs obtained from post Hartree-Fock $a b$ initio CASSCF and DFT methods, trying to highlight the origins of their differences. Furthermore, a comparison between a pure and hybrid exchange-correlation functional was performed to corroborate or to contradict the SD differences observed in the Fe" nitrosyl complex, using the same functionals as Boguslawski et al. ${ }^{[13]}$

\section{Source function spin density}

In 1998, Bader and Gatti introduced the SF descriptor, demonstrating that the ED at any point $\boldsymbol{r}, \rho(\boldsymbol{r})$, is caused by a local source (LS) function operating at all the other point $\boldsymbol{r}^{\prime}$ of the system ${ }^{[17]}$

$\rho(\boldsymbol{r})=\int_{R^{3}} \mathrm{LS}\left(\boldsymbol{r}, \boldsymbol{r}^{\prime}\right) d \boldsymbol{r}^{\prime}=\int_{R^{3}}-\frac{1}{4 \pi} \frac{\nabla^{2} \rho\left(\boldsymbol{r}^{\prime}\right)}{\left|\boldsymbol{r}-\boldsymbol{r}^{\prime}\right|} d \boldsymbol{r}^{\prime}$.

The LS function depends on the ED Laplacian $\nabla^{2} \rho\left(\boldsymbol{r}^{\prime}\right)$ evaluated at the point $\boldsymbol{r}^{\prime}$ and on the distance between this point and the one where $\rho(\boldsymbol{r})$ is reconstructed. This simple expression highlights a cause-effect relationship linking all the points of the system, where $\nabla^{2} \rho\left(\boldsymbol{r}^{\prime}\right)$ is the cause, $\rho(\boldsymbol{r})$ the effect and $\left(4 \pi\left|r-r^{\prime}\right|\right)^{-1}$ is a Green function that works as a weighting factor. When the whole space is partitioned according to the QTAIM definition of an atomic basin, ${ }^{[19]}$ that is, the region of space bounded by zero-flux surfaces (ZFSs) of $\nabla \rho(\boldsymbol{r})$, the integral over $R^{3}$ can be expressed as the sum of LS integrated inside all the QTAIM atoms $\Omega$. In this way, it is possible to express the reconstructed $\rho(\boldsymbol{r})$ in terms of atomic contributions, which provides precious chemical insights and an easy to grasp picture of the role played by the various atoms in determining the ED in a specific point of the space. When the LS is integrated inside a basin $\Omega$ it is called its Source Function $S$

$$
\rho(\boldsymbol{r})=\int_{R^{3}} \mathrm{LS}\left(\boldsymbol{r}, \boldsymbol{r}^{\prime}\right) d \boldsymbol{r}^{\prime}=\sum_{\Omega} \int_{\Omega} \mathrm{LS}\left(\boldsymbol{r}, \boldsymbol{r}^{\prime}\right) d \boldsymbol{r}^{\prime}=\sum_{\Omega} \mathrm{S}(\boldsymbol{r}, \Omega) .
$$

The SF descriptor can be easily extended to the SD case just replacing the scalar field $\rho(\boldsymbol{r})$ with $s(\boldsymbol{r})$ and $\nabla^{2} \rho\left(\boldsymbol{r}^{\prime}\right)$ with $\nabla^{2} s\left(\boldsymbol{r}^{\prime}\right)$

$$
s(\boldsymbol{r})=\int_{R^{3}} \mathrm{LS}_{\mathrm{s}}\left(\boldsymbol{r}, \boldsymbol{r}^{\prime}\right) d \boldsymbol{r}^{\prime}=\sum_{\Omega} \int_{\Omega}-\frac{1}{4 \pi} \frac{\nabla^{2} s\left(\boldsymbol{r}^{\prime}\right)}{\left|\boldsymbol{r}-\boldsymbol{r}^{\prime}\right|} d \boldsymbol{r}^{\prime}=\sum_{\Omega} S_{\mathrm{s}}(\boldsymbol{r}, \Omega) .
$$

The space partitioning is performed, also in this case, using the $\nabla \rho(\boldsymbol{r})$ ZFSs to maintain the definition of QTAIM atoms and the related SF contributions. A partitioning scheme associated to $\nabla s(\boldsymbol{r})$ is also possible and interesting, but the interpretation of the resulting basins and $S_{s}$ is more complicate. We are presently investigating this alternative strategy on very simple compounds and results will be reported in a future publication.

The $L S$ for the spin density $L S_{s}$ assumes the form

$$
\mathrm{LS}_{s}\left(\boldsymbol{r}, \boldsymbol{r}^{\prime}\right)=-\frac{1}{4 \pi} \frac{\nabla^{2} s\left(\boldsymbol{r}^{\prime}\right)}{\left|\boldsymbol{r}-\boldsymbol{r}^{\prime}\right|}=-\frac{1}{4 \pi} \frac{\nabla^{2}\left[\rho_{\alpha}\left(\boldsymbol{r}^{\prime}\right)-\rho_{\beta}\left(\boldsymbol{r}^{\prime}\right)\right]}{\left|\boldsymbol{r}-\boldsymbol{r}^{\prime}\right|}=\frac{\nabla^{2} \rho_{\beta}\left(\boldsymbol{r}^{\prime}\right)-\nabla^{2} \rho_{\alpha}\left(\boldsymbol{r}^{\prime}\right)}{4 \pi\left|\boldsymbol{r}-\boldsymbol{r}^{\prime}\right|}
$$

The $\nabla^{2} \rho\left(\boldsymbol{r}^{\prime}\right)$ and $\nabla^{2} s\left(\boldsymbol{r}^{\prime}\right)$ scalar fields dilute and concentrate in quite different ways throughout the molecular space and so lead to quite different atomic sources. ${ }^{[1,15]}$ Furthermore, at variance with the case of the ED, concentration or dilution of both $\alpha$ and $\beta$ components do not ensure a positive or negative $L_{S}$ because SD is defined as the difference between the $\rho_{\alpha}$ and $\rho_{\beta}$ density. For this reason, it is the relative weight of the $\alpha$ and $\beta$ terms that matters. In the former case, when the $\alpha$ component is more concentrated, that is, $\left|\nabla^{2} \rho_{\alpha}\left(\boldsymbol{r}^{\prime}\right)\right|>\left|\nabla^{2} \rho_{\beta}\left(\boldsymbol{r}^{\prime}\right)\right|$, the $\mathrm{LS}_{\mathrm{s}}$ in that point will be positive and it contributes to increase the SD in any other point $\boldsymbol{r}$, vice versa when $\left|\nabla^{2} \rho_{\alpha}\left(\boldsymbol{r}^{\prime}\right)\right|<\left|\nabla^{2} \rho_{\beta}\left(\boldsymbol{r}^{\prime}\right)\right|$ the $\mathrm{LS}_{\mathrm{s}}$ will be negative, decreasing the value of $s(\boldsymbol{r})$. Points or region with $\mathrm{LS}_{\mathrm{s}}>0$ $\left(\nabla^{2} s<0\right)$ will then produce a so-called $\alpha$ effect (an increasing of the $\rho_{\alpha}$ component in the point $\boldsymbol{r}$ ), on the contrary $\mathrm{LS}_{\mathrm{s}}<0$ $\left(\nabla^{2} s>0\right)$ will yield a $\beta$ effect. A more detailed discussion about this can be found in Gatti et al. paper published in 2015. ${ }^{[15]}$ 
The magnetic and relaxation (or reaction) decomposition of the SF values helps to ease their interpretation. The former term is related to the distribution of the unpaired $\alpha$ electrons, while the latter is due to all the other $\alpha$ and $\beta$ electrons. The magnetic density can be easily obtained by diagonalizing the first-order density matrix and by selecting those orbitals with occupation number equal or very close to one. Although the magnetic contribution is only due to $\alpha$ electrons that generate a positive total SD distribution everywhere, magnetic $\mathrm{LS}_{\mathrm{s}}$ may have both $\alpha$ and $\beta$ effects, depending on the sign of the magnetic density Laplacian. The relaxation term integrates over the whole space to zero, but locally can assume $s(\boldsymbol{r}) \neq 0$ values and its $\mathrm{LS}_{\mathrm{s}}$ can enhance or counteract the effect of the magnetic term. ${ }^{[11,15]}$

Atomic SF contributions are usually analyzed in terms of percentage contributions at a specific RP, expressing the ability of an atom or a group of atoms to determine the density (in this case SD) in this point ${ }^{[18]}$

$$
\mathrm{S}_{\mathrm{s}} \%(\mathrm{RP}, \Omega)=\frac{\mathrm{S}_{\mathrm{s}}(\mathrm{RP}, \Omega)}{\mathrm{s}(\mathrm{RP})} \cdot 100
$$

However, as mentioned in the introduction, the choice of a meaningful RP for the SF evaluation is not trivial in the case of the SD scalar field. To overcome this issue, we recently extended the SF analysis to an N-dimensional (ND) grid of RPs, by which it is possible to partially reconstruct density along a line $(N=1)$, in a plane $(N=2)$ or in a volume $(N=3)$ using a limited subset of atoms

$$
s\{\Omega, \text { subset }\}(\boldsymbol{r})=\sum_{\Omega, \text { subset }} \int_{\Omega} \mathrm{LS}_{\mathrm{s}}\left(\boldsymbol{r}, \boldsymbol{r}^{\prime}\right) d \boldsymbol{r}^{\prime}=\sum_{\Omega, \text { subset }} \mathrm{S}_{\mathrm{s}}(\boldsymbol{r}, \Omega) .
$$

When the subset includes all the atoms, the reconstructed SD should be equal to the total SD if no numerical errors are present.

These $\mathrm{N}$-dimensional SF analyses enable one to have a clearer picture of the role of a given subset of atoms in defining the SD in a selected region. Through this approach, it is possible to verify which zones are the most important in the spin information transmission and which atoms contribute more. Moreover, atomic SF partial reconstruction provides further insights on the origin of the discrepancies found in the SDs obtained from different theoretical methods. ${ }^{[11]}$ In this work, we will use both the $\mathrm{S}_{\mathrm{s}} \%$ analysis adopting the conventional ball-and-stick representation and the 2D SF partial reconstructed SD maps. ${ }^{[11,15]}$

To conclude this section, it is beneficial to remind that the information obtained from the SF descriptor is only a function of the total SD and is independent from any model through which it is expressed.

\section{Structural and magneto-structural information}

The azido group $\mathrm{N}_{3}^{-}$is one of the most used magnetic coupler in molecular magnets and it is classifiable as a non-innocent ligand. ${ }^{[21]}$ The $\mathrm{N}_{3}^{-}$is able to couple metallic centers in two

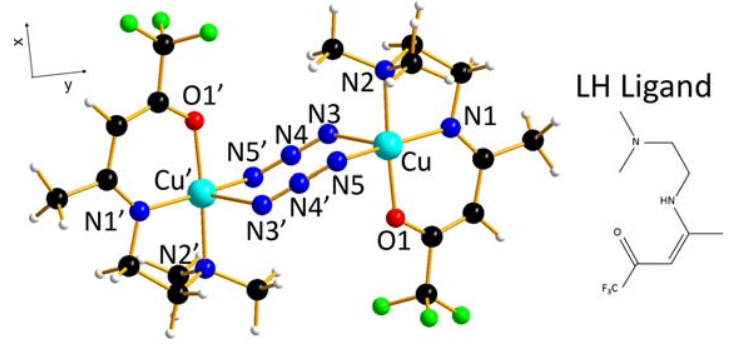

Figure 1. Molecular structure of the centrosymmetric end-end (EE) dicopper azido complex (left) and LH ligand scheme (right). The bond distances of the atoms coordinating the $\mathrm{Cu}^{\text {II }}$ cation are: $\mathrm{Cu}-\mathrm{N} 1=1.961 \AA$; $\mathrm{Cu}-\mathrm{N} 2=2.035 \AA ; \mathrm{Cu}-\mathrm{N} 3=2.356 \AA ; \mathrm{Cu}-\mathrm{N} 5=2.000 \AA ; \mathrm{Cu}-\mathrm{O} 1=1.927 \AA$. Atoms color code is: light blue (copper), green (fluorine), red (oxygen), blue (nitrogen), black (carbon), and white (hydrogen). [Color figure can be viewed at wileyonlinelibrary.com]

different ways: (i) through one terminal $\mathrm{N}$ atom ( $\mu-1,1 \mathrm{EO}$ coordination) or (ii) through the two terminal $\mathrm{N}$ atoms $(\mu-1,3 \mathrm{EE}$ coordination). The coordination geometry can be symmetric, when the $\mathrm{Cu}-\mathrm{N}$ bonds are equivalent, or asymmetric, when there is one short and one long $\mathrm{Cu}-\mathrm{N}$ bond.

In our previous work, ${ }^{[11]}$ we have studied the SD distribution in a symmetric EO azido bridged dicopper molecular complex (chemical formula $\left[\mathrm{Cu}_{2}(t \text {-Bupy })_{4}\left(\mathrm{~N}_{3}\right)_{2}\right]^{2+}, t$-Bupy $=p$-tert-butylpyridine), whose molecular geometry has been taken from the $18 \mathrm{~K}$ crystal structure of its perchlorate salt, obtained by Aebersold et al. in $1998 .^{[20]}$ In this work, we report the SF analysis of the calculated SD of the asymmetric EE Cull azido complex (chemical formula $\mathrm{Cu}_{2} L_{2}\left(\mathrm{~N}_{3}\right)_{2}$, with ligand $L=1,1,1$ trifluoro-7-(dimethylamine)-4-methyl-5-aza-3-hepten-2-onato), whose $150 \mathrm{~K} \mathrm{X}$-ray geometry has been determined by Aronica et al. in 2007. ${ }^{[21]}$

Figure 1 shows the centrosymmetric molecular structure of the EE complex and of the protonated L ligand $(\mathrm{LH})$, where the atomic labels are the same as reported in the original paper. ${ }^{[21]}$

The $\mathrm{Cu}^{\text {Il }}$ cation has a square-pyramidal geometry coordination, where the atoms $\mathrm{N} 1, \mathrm{~N} 2$, and $\mathrm{O} 1$ of the L ligand and one of the terminal nitrogen (N5) of the azido moiety form the squarepyramidal base, while the other terminal N (N3) of the second azido bridge coordinates from the top. This coordination geometry is responsible for the $\mathrm{N}_{3}^{-}$coordination asymmetry. The azido nitrogen belonging to the square base (N5) forms a short bond with the $\mathrm{Cu}^{\prime \prime}$ cation $(\mathrm{Cu}-\mathrm{N} 5=2.000 \AA)$, while the other terminal $\mathrm{N}(\mathrm{N} 3)$ forms a long bond $(\mathrm{Cu}-\mathrm{N} 3=2.356 \AA)$. In both EE and EO complexes, the two $\mathrm{Cu}^{\text {Il }}$ cations exhibit a ferromagnetic (FM) coupling but with different extent: the asymmetric EE system has a $J=17 \mathrm{~cm}^{-1}$, while the coupling constant for the symmetric EO is $J=300 \mathrm{~cm}^{-1} \cdot[20,21]$ This weak FM coupling in the EE system is due to the square-pyramidal geometry around the $\mathrm{Cu}^{\prime \prime}$ cations, because while the N5 atom points toward the magnetic $d_{x^{2}-y^{2}}$ orbitals of the paramagnetic center, the other terminal nitrogen N3 faces the non-magnetic $d_{z^{2}}$ orbital of the other $\mathrm{Cu}^{\text {II }}$ cation.

\section{Computational Details}

Chemical structure of the $\mathrm{Cu}_{2} \mathrm{~L}_{2}\left(\mathrm{~N}_{3}\right)_{2}$ molecular system ${ }^{[21]}$ (EE azido complex) was kept fixed at the crystal geometry through 
all the calculations. Theoretical SD calculations of the triplet ground-state were performed in vacuo at different levels of theory using the Gaussian16 program suite. ${ }^{[22]}$ Hybrid unrestricted B3LYP (UB3LYP) and pure unrestricted BLYP (UBLYP) exchange-correlation functionals were used within the unrestricted DFT (UDFT) approach. These functionals were chosen analogously to those used by Boguslawski et al., ${ }^{[13]}$ to compare hybrid and pure exchange-correlation DFT Hamiltonian. All the obtained results were corrected through spin annihilation procedure, leading to almost zero spin contamination $\left(<S^{2}\right\rangle=2.000$ for both UB3LYP and UBLYP). CASSCF computations were performed using different active spaces. The smallest active space used includes six electrons and six orbitals $[C A S(6,6)]$, four occupied, and two virtual. To increase the quality of the multi-configuration wavefunction, we expand the active space taking into account 10 electrons in 10 orbitals [CAS(10,10)]. Starting guesses were taken from unrestricted Hartree-Fock (UHF) natural orbitals (NOs), selecting both $A_{\mathrm{g}}$ (total symmetric gerade) and $A_{\mathrm{u}}$ (total symmetric ungerade) symmetries. The two initial half-full NOs are mainly localized on the $\mathrm{Cu}^{\text {" }}$ cations and have a "d-like" shape, with some tails on the coordinated ligand heteroatoms. Azido and $L$ ligand " $\pi / \pi^{*}$-like" NOs were selected to complete the $(6,6)$ and $(10,10)$ active spaces. The magnetic part of the SD was obtained selecting those NOs with occupation numbers very close to one. The procedure used to obtain the SD was the same described in our previous works. ${ }^{[11,15]}$ Peintinger et al. pobTVZP basis set was used through all the calculations. ${ }^{[23]}$

Modified version of AIMPAC program package by BieglerKönig et al. ${ }^{[24]}$ (i-ii) and home-developed codes (iii) have been used to evaluate different properties: (i) EXTREMESPIN, to calculate SD and SD Laplacian values at relevant stationary points; (ii) SPINSF2016, to evaluate Bader's basins properties and to obtain the SF contributions at selected RPs of a $N$ dimensional grid (with $N=0,3$ ); (iii) PLOTDEN2016: to plot the contour 2D maps of $s, \nabla^{2} s$, and SF reconstructed partial SDs. The ZFSs of the basins were obtained using the "classical" $\nabla \rho(r)$ line gradients to preserve the QTAIM definition of atoms. $^{[19]}$ All the structures here reported were visualized using the Diamond program, ${ }^{[25]}$ while the 3D SD isosurfaces were plotted using VESTA software. ${ }^{[26]}$

\section{Results}

Effect of the active space size on the CASSCF spin populations

DFT functionals are largely used to obtain information on molecules and transition metal complexes. ${ }^{[13]}$ One of the reasons of this success is that it is possible to treat large compounds with relatively low computational efforts. However, the treatment of open-shell systems remains a big challenge for all the DFT functionals, as the SD distribution is in principle not required to define the ground state of a specific compound, but it is included as an additional parameter in the Hamiltonian. ${ }^{[27]}$ The classical exchange-correlation functionals, such as for example B3LYP, BP86, and M06, are not calibrated to correctly reproduce the SD of magnetic systems, and this obviously results in non-accurate distributions. ${ }^{[13]}$

$A b$ initio electron correlation methods are then fundamental to recover accurate SDs. CASSCF is one of the most used post Hartree-Fock methods to introduce static correlation. Unfortunately, at least two "Achilles heels" affect this method and limit its systematic use. The first problem concerns the size of the active space, because usually the $a b$ initio softwares are able to manage a limited number of electrons and orbitals due to the large computational efforts required. The second critical point is the choice of the active orbitals, which very often is arbitrary and not completely appropriate. Obviously, the larger is the active space chosen, the more accurate the correlation correction is, within the computational limits. Large active spaces may generally lead to convergence failures and practical recipes to define their most suited size have been put forth. ${ }^{[28]}$

As we briefly described above, in this work we used two active spaces: the $\operatorname{CAS}(6,6)$, with 6 electrons distributed in 6 orbitals, and the $\operatorname{CAS}(10,10)$, with 10 electrons in 10 orbitals. CAS $(6,6)$ active space was chosen analogously to what reported in the EO azido complex work, where we select the two "d-shape" singly occupied NOs located on the Cu" cation and the " $\pi$-like" NOs on the azido moiety. ${ }^{[11]}$ Here, we were however able to expand the $(6,6)$ active space up to $(10,10)$, including the " $\pi / \pi^{*}$ " NOs of the $L$ ligand. A further increment to $\operatorname{CAS}(14,14)$ was not possible due to technical limits. The selection of the proper UHF active NOs for CASSCF calculation, chosen as described in the Computational Details section, was easily performed, as they were HOMOs-LUMOs orbitals. A similar expansion of the active space to that adopted here for the EE system had not been possible for the EO azide because of symmetry issues. ${ }^{[11]}$

UHF method shows a high spin contamination effect $\left.\left(<S^{2}\right\rangle=2.0946\right)$ and annihilation procedure was necessary to recover the correct spin state (spin-annihilated final state $\left.\left\langle S^{2}\right\rangle=2.0045\right)$. Energy gain relative to the UHF wavefunction obviously increases with the size of the CASSCF active space. It amounts to -0.0575 au for the $\operatorname{CAS}(6,6)$ and almost doubles $(-0.1023 \mathrm{au})$ for the $\operatorname{CAS}(10,10)$ model wavefunction, while the number of configurations increase from 225 to 44,100. Energy data of all the wavefunctions used in this article are reported in the Supporting Information (Table S1).

The significant energy gain from $\operatorname{CAS}(6,6)$ to $\operatorname{CAS}(10,10)$ implies that the added starting NOs are important for the correct description of the system and that a reasonable convergence is not reached at the $\operatorname{CAS}(6,6)$ level. However, a full convergence cannot be ensured even at the $\operatorname{CAS}(10,10)$ level, yet the rapid growth of the configuration numbers and the technical limits do not allow us to further increase the active space. Magnetic orbitals were selected based on occupation numbers. These latter were negligibly different from one at the CAS $(6,6)$ level and equal to almost one (1.032 and $0.977 e$ ) at the $\operatorname{CAS}(10,10)$ level.

The UHF and CASSCF QTAIM electron net charges, $q(\Omega)$, and electron spin populations, $\operatorname{SP}(\Omega)$, for the $\mathrm{Cu}$ atom, the azido moiety (N3, N4, and N5), and some L atoms (N1, N2, and O1) are reported in Table 1. 
Table 1. QTAIM electron net charges, $q(\Omega)$, and electron spin populations, $\mathrm{SP}(\Omega)$, for selected atoms $\Omega$ at three different levels of theory: UHF, $\operatorname{CAS}(6,6)$, and $\operatorname{CAS}(10,10)$

\begin{tabular}{|c|c|c|c|c|c|c|}
\hline \multirow[b]{2}{*}{ Basin } & \multicolumn{3}{|c|}{$q(\Omega)$} & \multicolumn{3}{|c|}{$\operatorname{SP}(\Omega)$} \\
\hline & UHF & $\operatorname{CAS}(6,6)$ & $\operatorname{CAS}(10,10)$ & UHF & $\operatorname{CAS}(6,6)$ & $\operatorname{CAS}(10,10)$ \\
\hline $\mathrm{Cu}$ & 1.459 & 1.463 & 1.460 & 0.891 & 0.909 & 0.821 \\
\hline N1 & -1.440 & -1.441 & -1.362 & 0.033 & 0.020 & 0.057 \\
\hline N2 & -1.209 & -1.210 & -1.212 & 0.028 & 0.021 & 0.019 \\
\hline N3 & -0.218 & -0.204 & -0.196 & -0.086 & 0.002 & 0.023 \\
\hline N4 & -0.201 & -0.248 & -0.259 & 0.009 & 0.003 & 0.003 \\
\hline N5 & -0.401 & -0.370 & -0.357 & 0.109 & 0.017 & 0.034 \\
\hline 01 & -1.329 & -1.330 & -1.342 & 0.021 & 0.020 & 0.019 \\
\hline$\Sigma^{\prime}$ & -3.339 & -3.339 & -3.269 & 0.114 & 0.083 & 0.155 \\
\hline
\end{tabular}

The sum of $q(\Omega)$ or $\operatorname{SP}(\Omega)$ values over the selected atoms, except $\mathrm{Cu}$, is denoted as $\Sigma$ '. Space partitioning has been performed according to $\nabla \rho(\mathrm{r})$ zero-flux surfaces.

Hereinafter, in the whole text the term $\mathrm{Cu}$ "l cation(s) will be replaced by $\mathrm{Cu}$ atom(s) when properties of the QTAIM basins or of those derived from Mulliken's partitioning are discussed.

The first remark one can extract looking at the data reported in Table 1 is that the net charges are more stable than the spin populations (SP) against model change. $\mathrm{Cu}$ net charge remains more or less unchanged moving from the UHF method $(1.459 e)$ to $\operatorname{CAS}(6,6)(1.463 e)$ and to $\operatorname{CAS}(10,10)$ $(1.460$ e) models, while the SP of the $\mathrm{Cu}$ atom raises first from 0.891 to $0.909 e$ (UHF and $\operatorname{CAS}(6,6)$, respectively), then it decreases to $0.821 e$ in the $\operatorname{CAS}(10,10)$ model. This large fluctuation is intrinsic in the SD definition, as a compensation of $\alpha$ and $\beta$ components of the $\rho$ (leading to an increment of the $\alpha$ and a similar reduction of the $\beta$ population) recovers the same total population and net charge, but results in a largely different SP $\left(\mathrm{SP}(\Omega)=\int_{\Omega}\left(\rho_{\alpha}-\rho_{\beta}\right) d r\right)$. However, the increase of one component at the expense of the other has a physical significance and may be an indicator of the shortcomings of a model wavefunction (see infra). The most evident SP changes occur on the azido moiety. The total SP of this group little varies from the UHF value of $0.032 e$ to a value of 0.022 and 0.060 e for the $\operatorname{CAS}(6,6)$ and $\operatorname{CAS}(10,10)$ models, respectively. But what are relevant are the population variations within the group. The CAS $(6,6)$ model smooths the SP distribution of the $\mathrm{N}$ atoms. UHF predicts a high positive SP located on the shortbonded N5 atom (0.109 e), while the long-bonded N3 shows a negative population of similar magnitude $(-0.086 e)$, pointing to a spin polarization mechanism within the ligand. The CAS $(6,6)$ method contradicts this description, predicting a much smaller SP on the N5 atom (0.017) and a positive, close to zero, SP on the N3. Here, the polarization mechanism disappears, in favor of a spin delocalization mechanism. Both UHF and CAS $(6,6)$ methods predict a small positive SP on the N4, the central atom of the azido bridge. On average, the $\operatorname{CAS}(6,6)$ $\mathrm{SP}$ on the $\mathrm{L}$ atoms is smaller in magnitude than at the UHF level. When the active space is extended from $(6,6)$ to $(10,10)$, the overall $\alpha$-spin delocalization toward both the azido moieties and the $\mathrm{L}$ ligands grows up. This is summarized by the sum $\Sigma^{\prime}$ of the SPs of the atoms listed in Table 1, except $\mathrm{Cu}$, which raises from $0.083 e$ in $\operatorname{CAS}(6,6)$ to $0.155 e$ in the
CAS $(10,10)$ model. Such an increase is probably due to the introduction in the active space of the $\pi / \pi^{*}$ NOs of the $L$ ligands, which returns a more flexible model and allows a greater $\alpha$-spin delocalization.

Figure 2 shows 3D SD isovalue surfaces plots for the UHF and CAS calculations. Although $\operatorname{CAS}(10,10)$ and $\operatorname{CAS}(6,6)$ calculations do not completely agree from a quantitative point of view, they yield qualitatively similar SD distribution, while UHF ones greatly differ.

UHF predicts extended negative regions (colored in light blue) around the $\mathrm{Cu}^{\prime \prime}$ cations and on N4 (N4') and N3 (N3') atoms of the azido moieties, which are completely or partially missing in the Multi Configuration Self Consistent Field (MCSCF) calculations. Positive and negative SD accumulations alternating within the azido moieties indicate spin polarization, which complies with the large and opposite SPs reported in Table 1. The presence of positive and negative zones on the azido central N4 justifies its almost zero SP. Small, but not negligible, negative SD regions can be also found on the $L$ carbon atoms. In the $\operatorname{CAS}(6,6)$ isovalue map, the negative regions
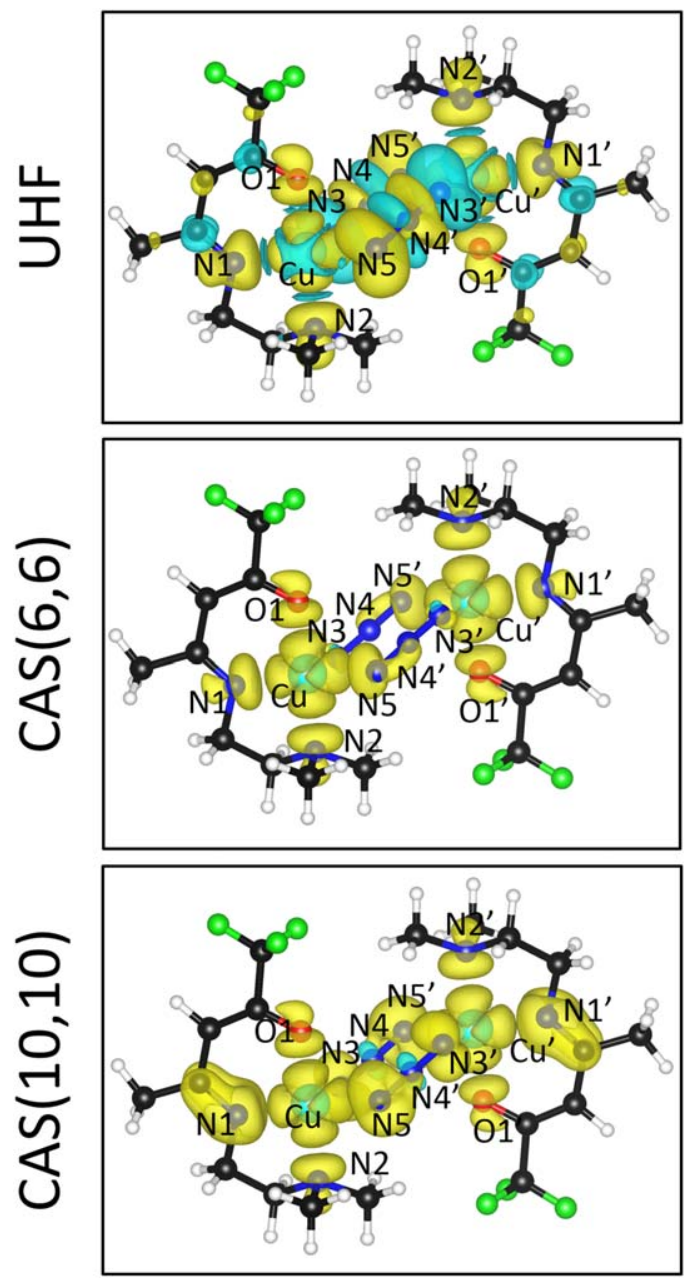

Figure 2. Three-dimensional spin density isovalue surfaces plots for the UHF, CAS(6,6), and $\operatorname{CAS}(10,10)$ calculations. Isovalue levels are fixed at 5 $\times 10^{-4}$ e. Positive values are reported in yellow, negative values in light blue. These isovalue surfaces plots are reproduced using VESTA software. ${ }^{[26]}$ [Color figure can be viewed at wileyonlinelibrary.com] 
Table 2. Spin density populations, SPs, in the end-end azido $\mathrm{Cu}^{\text {Il }}$ dinuclear complex.

\begin{tabular}{|c|c|c|c|c|}
\hline$\Omega$ & $\operatorname{SP}(\Omega)^{[a]}$ & $\mathrm{SP}_{\mathrm{Mul}}(\Omega)^{[\mathrm{a}]}$ & $\operatorname{SP}_{\text {Aronica }}(\Omega)^{[\mathrm{b}]}$ & $\operatorname{SP}_{\mathrm{PND}}(\Omega)^{[\mathrm{Cc}}$ \\
\hline $\mathrm{Cu}$ & $0.8210 .640 / 0.528)$ & $0.839(0.650 / 0.534)$ & $0.806(0.571)$ & $0.719[6]$ \\
\hline N1 & $0.057(0.069 / 0.078)$ & $0.053(0.069 / 0.079)$ & $0.028(0.100)$ & $0.044[5]$ \\
\hline N2 & $0.019(0.078 / 0.089)$ & $0.015(0.077 / 0.090)$ & $0.021(0.113)$ & $0.076[6]$ \\
\hline N3 & $0.023(0.065 / 0.091)$ & $0.022(0.073 / 0.100)$ & $0.040(0.113)$ & $0.033[7]$ \\
\hline N4 & $0.003(0.005 / 0.010)$ & $0.005(-0.001 / 0.002)$ & $0.004(-0.022)$ & $0.004[5]$ \\
\hline N5 & $0.034(0.042 / 0.066)$ & $0.031(0.038 / 0.064)$ & $0.039(0.044)$ & $0.029[6]$ \\
\hline 01 & $0.019(0.067 / 0.083)$ & $0.013(0.065 / 0.082)$ & $0.044(0.077)$ & $0.043[5]$ \\
\hline$\Sigma_{\text {azide }}$ & $0.060(0.112 / 0.167)$ & $0.058(0.110 / 0.166)$ & $0.083(0.135)$ & $0.066[18]$ \\
\hline$\Sigma_{\mathrm{L}}$ & $0.095(0.214 / 0.250)$ & $0.081(0.211 / 0.251)$ & $0.093(0.290)$ & $0.163[16]$ \\
\hline \multicolumn{5}{|c|}{$\begin{array}{l}\text { The atoms labels are the same of Figure } 1 \text {. The sum of } \mathrm{SP}(\Omega) \text { values over the atoms belonging to the } \mathrm{L} \text { ligand }(\mathrm{N} 1, \mathrm{~N} 2 \text {, and } \mathrm{O} 1) \text { is denoted as } \Sigma_{\mathrm{L}} \text {, while } \\
\text { the sum of the corresponding values over the azido } \mathrm{N} \text { atoms }(\mathrm{N} 3, \mathrm{~N} 4 \text {, and } \mathrm{N} 5) \text { is denoted } \Sigma_{\text {azide. }} \text { [a] Data from the CAS(10,10) wavefunction (UB3LYY } \\
\text { UBLYP results in parentheses). SP( }(\Omega) \text { : QTAIM partitioning scheme, SP Mul }(\Omega) \text { : Mulliken's partitioning. [b] DDCI-3 Mulliken's spin populations from Table } 5 \\
\text { of Ref. [21]. DFT populations in parentheses. [c] Polarized Neutron Diffraction (PND) data from Table } 5 \text { of Ref. [21]. Estimated standard deviation in } \\
\text { brackets. }\end{array}$} \\
\hline
\end{tabular}

completely disappear except for small lobes around the N3 atom. Here, we can clearly see that the opposite spin zones predicted by the UHF just annihilate each other, leading to a contraction of the positive spin envelope located on the N5 and to a more smoothed distribution on the whole azido ligand. Beyond these differences, both UHF and CAS $(6,6)$ maps show the same positive four-lobed SD distribution around the $\mathrm{Cu}^{\prime \prime}$ cations and similar SD in the region of the $L$ heteroatoms. The $\operatorname{CAS}(10,10)$ spin isovalue surfaces are very much alike those for the smaller active space $(6,6)$ except few noticeable differences: (i) the positive regions located on the N3 and N5 atoms are bigger, in accordance to the values reported in Table 1; (ii) small negative lobes appear on the central N4 atom; and (iii) the N1 atom shows a more delocalized SD isosurface. Analogous 3D SD isovalue surfaces plots for the two DFT functional adopted, UB3LYP and UBLYP, are shown in Figure S1 of the Supporting Information and compared with the $\operatorname{CAS}(10,10)$ ones. From now on, only the $\operatorname{CAS}(10,10)$ results will be used in the analysis of the SD distribution of the EE azido complex. $\operatorname{CAS}(10,10)$ is also taken as the reference correlated model for comparison with DFT results (see next section).

\section{CAS $(10,10)$ versus DFT and PND spin populations}

CAS $(10,10)$ SP for the atoms listed in Table 1 are compared in Table 2 with those obtained at the DFT level of theory (present work) and those reported in Table 5 of Ref. [21] (from DDCI-3/DFT computations and PND data refinement). As the DDCI-3/DFT results refer to Mulliken's populations, $\operatorname{CAS}(10,10)$ and DFT Mulliken's SPs, $\operatorname{SP}_{\text {Mul }}(\Omega)$, are also listed in Table 2, for the sake of a fair comparison. QTAIM atomic charges at CAS $(10,10)$ and at the DFT level of theory adopted in the present work are reported in the Supporting Information (Table S2).

Similarly to what was found in previous works and in the preceding paragraphs, populations are strongly dependent on the computational method and on the DFT functional used. ${ }^{[11,15]}$ The not negligible differences within the DFT functionals reside in the nature of the DFT theory, both the electronic energy and the electronic observables are herein defined by the ED distribution only. As stated before, SD is not in principle needed to define the exchange-correlation functionals, but it is a pure additional variable in the Hamiltonian.

$\operatorname{CAS}(10,10)$ calculation estimates that the $82 \%$ of the total 2 e SP resides on the $\mathrm{Cu}$ basins, the $6 \%$ is located on the azido moieties, while the $10 \%$ is concentrated on the heteroatoms coordinating the metal centers. Mulliken's population are similar to Bader's ones, resulting in $84 \%, 6 \%$, and $8 \%$ percentage, respectively. These data are very close to those obtained by Aronica et al. using a DDCl-3 method (81\%, 8\%, and $9 \%$ percentage, respectively). ${ }^{[21]}$ DFT predictions are instead completely different. UB3LYP QTAIM (Mulliken's) populations indicate that only $64 \%(65 \%)$ of the two unpaired electrons reside on the $\mathrm{Cu}$ atoms, while UBLYP retrieves for them only the $53 \%$ of the whole SP. These values are decisively lower than those found for the $\operatorname{CAS}(10,10)$, confirming that DFT calculations largely exaggerate the $\alpha$-spin delocalization toward the ligands. ${ }^{[11,13]}$ This interpretation is strengthened if one looks at the SPs on the azido and ligand atoms. For UB3LYP, an overall $11 \%$ of QTAIM SP lies on the $\mathrm{N}_{3}^{-}$bridges and a $21 \%$ of it is located onto ligand heteroatoms. The corresponding numbers for the UBLYP functional are $17 \%$ and $25 \%$, respectively. At least for this compound, pure UBLYP exchangecorrelation functional has a higher tendency to delocalize the two unpaired electrons than hybrid UB3LYP. These outcomes reveal an opposite trend respect to what Boguslawski et al. found for the Fe" nitrosyl complex, where pure UBLYP functional shows smaller differences than hybrid UB3LYP functional when compared to CASSCF results. ${ }^{[13]}$ This fact seems to suggest that no general rules about the accuracy of DFT functionals, according to their different nature, in predicting SD can be stated before further investigations. PND results by Aronica et al. are intermediate between the $\operatorname{CAS}(10,10)$ and the UB3LYP outcomes, estimating that the $72 \%$ of the SP lies on the $\mathrm{Cu}$ atoms, the $7 \%$ on the azido moieties and the $16 \%$ on the $L$ ligand heteroatoms. ${ }^{[21]}$ By refining the parameters of $a$ spin-split multipolar model against X-ray and PND data, Deutsch et al. ${ }^{[3]}$ found a SP percentage on the $\mathrm{Cu}$ atoms of $74 \%$, quite close to the value $(72 \%)$ predicted by Aronica ${ }^{[21]}$ (252 and 125 magnetic structure factors were respectively used in the refinements). From a qualitatively viewpoint, all 
Table 3. Net charges $q(\Omega)$ and electron spin populations SP $(\Omega)$ in the EE complex for DFT wavefunctions, evaluated using the CAS $(10,10)$ QTAIM atomic boundaries.

\begin{tabular}{|c|c|c|c|c|c|c|c|c|}
\hline \multirow[b]{2}{*}{$\Omega$} & \multicolumn{2}{|c|}{$q(\Omega)$} & \multicolumn{2}{|c|}{$\Delta q(\Omega)$} & \multicolumn{2}{|c|}{$s(\Omega)$} & \multicolumn{2}{|c|}{$\Delta s(\Omega)$} \\
\hline & UB3LYP & UBLYP & UB3LYP & UBLYP & UB3LYP & UBLYP & UB3LYP & UBLYP \\
\hline $\mathrm{Cu}$ & 1.279 & 1.233 & 0.144 & 0.191 & 0.640 & 0.528 & 0.000 & 0.000 \\
\hline N1 & -1.331 & -1.309 & -0.252 & -0.308 & 0.069 & 0.078 & 0.000 & 0.000 \\
\hline N2 & -1.128 & -1.105 & -0.238 & -0.285 & 0.078 & 0.089 & 0.000 & 0.000 \\
\hline N3 & -0.177 & -0.164 & 0.050 & 0.058 & 0.065 & 0.090 & 0.000 & -0.001 \\
\hline N4 & -0.257 & -0.269 & -0.123 & -0.147 & 0.005 & 0.011 & 0.000 & 0.001 \\
\hline N5 & -0.324 & -0.300 & 0.028 & 0.028 & 0.042 & 0.066 & 0.000 & 0.000 \\
\hline$\Sigma_{\text {azide atoms }}$ & -0.758 & -0.733 & -0.045 & -0.061 & 0.112 & 0.167 & 0.000 & 0.000 \\
\hline $\mathrm{O} 1$ & -1.221 & -1.190 & -0.147 & -0.184 & 0.067 & 0.084 & 0.000 & 0.001 \\
\hline
\end{tabular}

the adopted methods agree on the SP magnitude of the central nitrogen of the azido moiety (N4), that is very close to zero, while they are discordant on the relative SP weights of the N3 and N5 atoms, those bonded to Cull cation.

A deeper insight on the nature of the remarkable differences between the Cu SD population of the CASSCF and DFT methods can be obtained by an in tandem exam of their EDs and SDs properties. Indeed the question may arise whether the enhanced delocalization of the unpaired ED to the ligands is but a consequence of the excessive electron delocalization typical of DFT (while HF methods behave oppositely). ${ }^{[29]}$ As shown in Supporting Information Table S2, the Cu net charges predicted by the DFT functionals $(q=1.135 / 1.042$ e for UB3LYP/UBLYP) are significantly lower than the CAS $(10,10)$ estimate $(q=1.460 e)$, meaning that more electron population lies in the $\mathrm{Cu}$ DFT basin relative to the $\operatorname{CAS}(10,10)$ one. The increased distances between the $\mathrm{Cu}$ nucleus and the bond critical points (BCPs) with bonded neighboring atoms and the expansion of the $\mathrm{Cu}$ basin volume (evaluated using a $\rho$ cutoff equal to $10^{-3}$ e) reveal that DFT, in particular the UBLYP exchange-correlation functional, overestimates the $\mathrm{Cu}$ basin size and enhances covalency of Cu-ligand bonds (as charge transfer is reduced). Taking the $\operatorname{CAS}(10,10)$ results as a reference, UB3LYP shows a variation equal to +0.05 au for the $\mathrm{Cu}$ BCPs average distance and to +7.6 au for the $\mathrm{Cu}$ volume, while the UBLYP corresponding enhancements are +0.07 au and $+10.1 \mathrm{au}$, respectively. All these mentioned changes, relative to the $\operatorname{CAS}(10,10)$ estimates, are caused by the exaggerated electron delocalization inherent to DFT. To help to disentangle, if ever possible, ED from electron SD DFT effects, we have evaluated DFT atomic QTAIM net charges and SPs using the $\operatorname{CAS}(10,10)$ atomic boundaries. Results are listed in Table 3, showing a significant effect on net charges and a negligible one on the SPs. When the $\operatorname{CAS}(10,10)$ boundaries are adopted, the $\mathrm{Cu}$ atom becomes more positively charged and the $\mathrm{L}$ ligand heteroatoms all increase their negative net charge. The azide group also increases its electron population, becoming as a whole more negatively charged. SPs remain instead unaffected by the change of boundaries, implying either a compensation of the $\alpha$ - and $\beta$-ED contributions effects and/or, more likely, the inclusion/exclusion of regions where the SD is very low.
$\mathrm{SD}$ contour maps drawn in the least squares plane of the $\mathrm{Cu}-\mathrm{O}$ and of the three shorter $\mathrm{Cu}-\mathrm{N}$ bonds show that the DFT functionals predict a small negative SD region between the $\mathrm{Cu}$ nucleus and the bonded atoms (Fig. 3). The enhanced electron delocalization of DFT could be responsible for this spin polarization mechanism, which likely leads to the mentioned covalency increase of the $\mathrm{Cu}-\mathrm{X}$ bonds $(\mathrm{X}=\mathrm{N}, \mathrm{O})$. To make a step further in our attempt to untangle ED from electron SD DFT effects, we have also analyzed the ED and electron SD differences, relative to CASSCF, for the two DFT selected approaches. The total densities and their differences are pictorially illustrated in the least-square plane of the $\mathrm{Cu}-\mathrm{O}$ and of the three shorter $\mathrm{Cu}-\mathrm{N}$ bonds (Fig. 3).

Comparison of the ED maps visibly shows that both DFT approaches describe the $\mathrm{Cu}-\mathrm{N}$ bonds and the $\mathrm{Cu}-\mathrm{O}$ bond as more shared and covalent in nature, relative to $\operatorname{CAS}(10,10)$ method (consider for instance the shape of isodensity contours close to the $\mathrm{Cu}-\mathrm{N} 5 \mathrm{BCP})$. As already mentioned, this implies a less positively charged $\mathrm{Cu}$ atom and less electron rich $\mathrm{N}$ and $\mathrm{O}$ ligand atoms (Supporting Information Table S2; note that $\mathrm{N} 3$, the azide atom involved in the long $\mathrm{Cu}-\mathrm{N}_{\text {azide }}$ bond with the "doubly occupied" $\mathrm{d}_{\mathrm{z}^{2}}$ orbital behaves oppositely). The ED differences indeed show that, along the $\mathrm{Cu}$ ligand directions and relative to the two DFT approaches, the CAS method predicts less ED from the $\mathrm{Cu}$ nucleus to the $\mathrm{Cu}$ ligand $B C P$ and, vice versa, more ED from this $B C P$ to the ligand nuclei. Then, one notices some compensating mechanism close to the nuclei (for $\mathrm{Cu}$ also in the directions of the "doubly" occupied orbital $\mathrm{d}_{\mathrm{xy}}$ ) with an opposite charge transfer, relative to that observed along the Cu-ligand "bonding" regions. This fact may reasonably justify why $\mathrm{N} 5_{\text {azide }}$ undergoes only a small decrease of its net charge on going from CAS $(10,10)$ to DFT (Supporting Information Table S2).

The increase of covalency of $\mathrm{Cu}-\mathrm{N}$ interactions in DFT implies a decrease of the Cu SP and a consequent increase of the SP on ligands (compare $s(\boldsymbol{r})$ plots). This is clearly illustrated by the SD difference maps and, in particular, by the differences along the $\mathrm{Cu}$-ligands interactions. At the $\operatorname{CAS}(10,10)$ level, there is an higher SD from the $\mathrm{Cu}$ nucleus to the $\mathrm{Cu}$-ligand $B C P$ (so within the $\mathrm{Cu}$ basin) and a lower SD from almost this $\mathrm{BCP}$ up to the nuclei of the ligands (so within their basins), relative to DFT. 

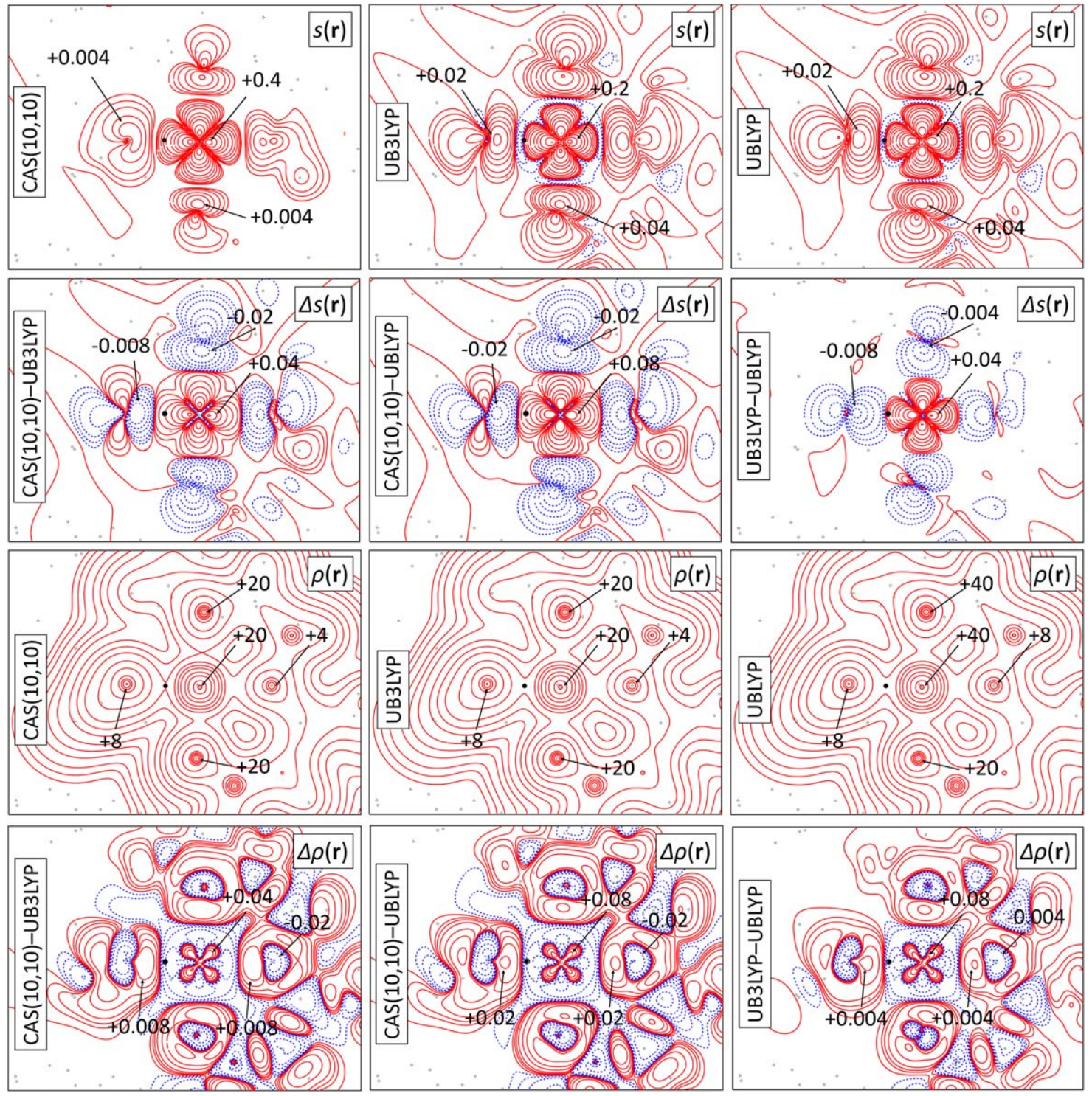

Figure 3. CAS $(10,10)$, UB3LYP, and UBLYP $2 \mathrm{D}$ contour plots of the spin density (top panels) and of the electron density (third row panels) in the leastsquare plane of the $\mathrm{Cu}-\mathrm{O}$ and of the three shorter $\mathrm{Cu}-\mathrm{N}$ bonds. In the second and fourth row panels, $[C A S(10,10)$ - DFT] and [DFT - DFT] difference electron and electron spin densities are shown. The position of the BCP of the $\mathrm{Cu}-\mathrm{N} 5$ bond, the short $\mathrm{Cu}$-azide bond, is denoted by a dot and refers to the portrayed density (row 1 and row 3). In the case of density differences, it refers to the CAS $(10,10)$ wavefunction. Positive and negative contours are portrayed as solid red and dotted blue lines, respectively. Contour maps are drawn at interval of $\pm(2,4,8) \cdot 10^{-n}, 0 \leq n \leq 4$ au. [Color figure can be viewed at wileyonlinelibrary.com]

As a global effect one expects that the lower electron population on $\mathrm{Cu}$ in $\operatorname{CAS}(10,10)$ should involve a preferential decrease of its $\beta$-electron over its $\alpha$-electron population relative to DFT. Vice versa, the increase of electron population on the ligands atoms linked to $\mathrm{Cu}(\mathrm{N} 1, \mathrm{~N} 2, \mathrm{~N} 5$, and $\mathrm{O} 1)$ is accompanied by a preferred increase of their $\beta$-electron over their $\alpha$-electron populations in $\operatorname{CAS}(10,10)$ relative to DFT. Data in Table 4 confirms this reasoning, especially for the $\mathrm{Cu}$ atom population change. In the case of the ligand atoms, the effect is less pronounced, although in the expected direction, because of the mentioned partly compensating effects close to the nuclei of the ligands.

Summarizing, the higher electron delocalization in DFT, causing a decreased charge separation between the $\mathrm{Cu}$ and the ligands, also implies an enhanced redistribution of the $\alpha$ spin excess on the $\mathrm{Cu}$ to the ligands. Such enhanced spin delocalization in DFT has been previously discussed in the literature. ${ }^{[30,31]}$

Spin density decomposition: total, magnetic, and relaxation spin densities maps

Spin density contour maps and their magnetic and relaxation components for the $\operatorname{CAS}(10,10)$ and DFT calculations, in the least-squares plane of the $\mathrm{Cu}-\mathrm{O}$ and of the three shorter $\mathrm{Cu}-\mathrm{N}$ bonds, are shown in Figure 4.

The unpaired electrons distribution in this plane of the $\mathrm{EE}$ azido complex recalls what was found in our previous work concerning the EO azido dicopper system. ${ }^{[11]}$ The total SD (left

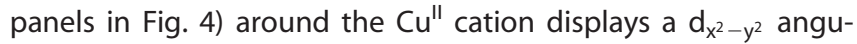
lar shape, where the four lobes point toward the atoms 
Table 4. $\alpha$ and $\beta$ populations $\left(N_{\alpha}\right.$ and $\left.N_{\beta}\right)$ in the EE complex for the $\operatorname{CAS}(10,10)$ and the DFT wavefunctions, along with their (CAS - DFT) changes $\Delta N_{\alpha}$ and $\Delta \mathrm{N}_{\beta}$.

\begin{tabular}{|c|c|c|c|c|c|c|c|c|}
\hline \multirow[b]{2}{*}{$\Omega$} & \multicolumn{2}{|c|}{ UB3LYP } & \multicolumn{2}{|c|}{ UBLYP } & \multicolumn{4}{|c|}{$\operatorname{CAS}(10,10)$} \\
\hline & $N_{\alpha}$ & $N_{\beta}$ & $N_{\alpha}$ & $N_{\beta}$ & $N_{\alpha}$ & $N_{\beta}$ & $\Delta N_{\alpha}(\text { UB3LYP; UBLYP })^{[a]}$ & $\Delta N_{\beta}(U B 3 L Y P ; \text { UBLYP })^{[\mathrm{a}}$ \\
\hline $\mathrm{Cu}$ & 14.25 & 13.61 & 14.24 & 13.71 & 14.18 & 13.36 & $-0.07 ;-0.06$ & $-0.27 ;-0.35$ \\
\hline N1 & 4.07 & 4.00 & 4.04 & 3.96 & 4.21 & 4.15 & $0.14 ; 0.17$ & $0.15 ; 0.19$ \\
\hline $\mathrm{N} 2$ & 3.98 & 3.91 & 3.95 & 3.87 & 4.12 & 4.10 & $0.14 ; 0.19$ & $0.17 ; 0.23$ \\
\hline N5 & 3.70 & 3.66 & 3.70 & 3.63 & 3.70 & 3.66 & $0.00 ; 0.00$ & $0.00 ; 0.03$ \\
\hline 01 & 4.57 & 4.50 & 4.54 & 4.46 & 4.68 & 4.66 & $0.11 ; 0.14$ & $0.16 ; 0.20$ \\
\hline
\end{tabular}

[a] $\Delta N_{\alpha}$ (UB3LYP; UBLYP) and $\Delta N_{\beta}$ (UB3LYP; UBLYP) are the populations changes $\left(N_{x, \text { CAS(10,10) }}-N_{x, \text { UB3LYP; }} N_{x, \text { CAS }(10,10)}-N_{x, \text { UBLYP }}\right)$ with $x=\alpha$ and $\beta$, respectively).

forming the base of the square-pyramid (N1, N2, N5, and O1). The fifth heteroatom bonded to the $\mathrm{Cu}^{\prime \prime}$ cation resides outside the plane, pointing to the $d_{z^{2}}$ orbital of the metal center. This evidence is true for all the used theoretical approaches. UB3LYP and UBLYP SD maps are qualitatively very similar. Both predict, as already anticipated in the previous section, a negative SD region in the outermost part of the $\mathrm{Cu}$ basin and a possible spin polarization mechanism between the magnetic center and the ligands. This feature is not observed in the CAS $(10,10)$ SD, where no polarization occurs in the selected plane. Comparison of the DFT and CAS innermost contour values on the ligand atoms, confirms that the former method exaggerates spin delocalization $(0.02 / 0.04 e$ and $0.004 / 0.008 e$ for DFT functionals and CASSCF, respectively), as already observed in Table 2 . The magnetic and relaxation SD decomposition enables us to clearly understand the differences between the methods and to better interpret their results. The CAS $(10,10)$ and the DFT (both UB3LYP and UBLYP functionals) magnetic contributions qualitatively agree one to each other. As expected, the magnetic contributions are the dominant ones in determining the total SDs, in particular the $d_{x^{2}-y^{2}}$ lobes around the $\mathrm{Cu}$ nucleus and the main part of the SDs located on the ligands. The different spin delocalization tendency discussed before for the total SDs is evident also for their magnetic components. The relaxation contributions predicted by the two main approaches are instead completely
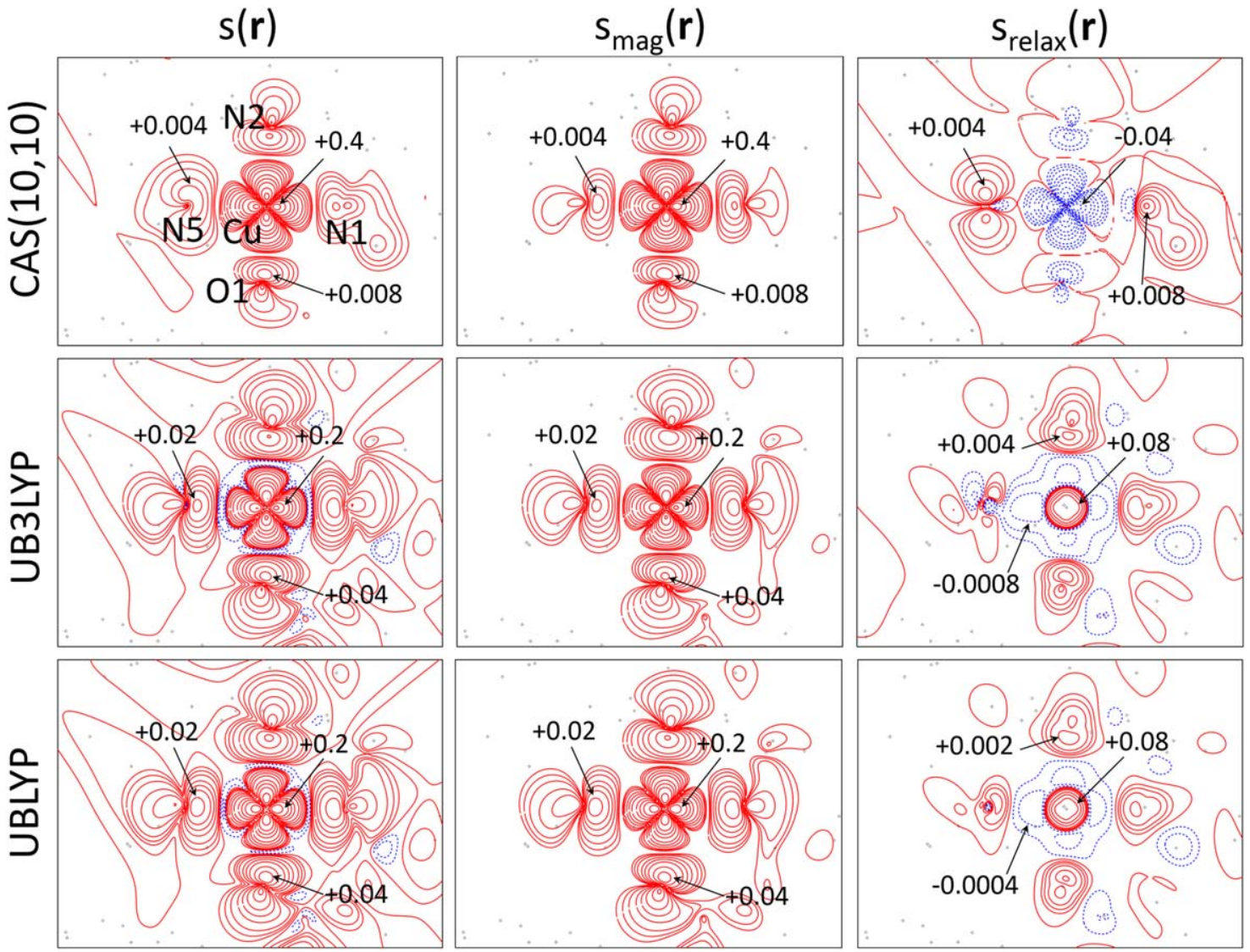

Figure 4. CAS(10,10) (first row), UB3LYP (second row), and UBLYP (third row) 2D contour plots of the total spin density (left) and of its magnetic (middle) and relaxation (right) terms in the least-square plane of the $\mathrm{Cu}-\mathrm{O}$ and of the three shorter $\mathrm{Cu}-\mathrm{N}$ bonds. Color codes, isovalue contours, and plane orientation are the same as in Figure 3. [Color figure can be viewed at wileyonlinelibrary.com] 


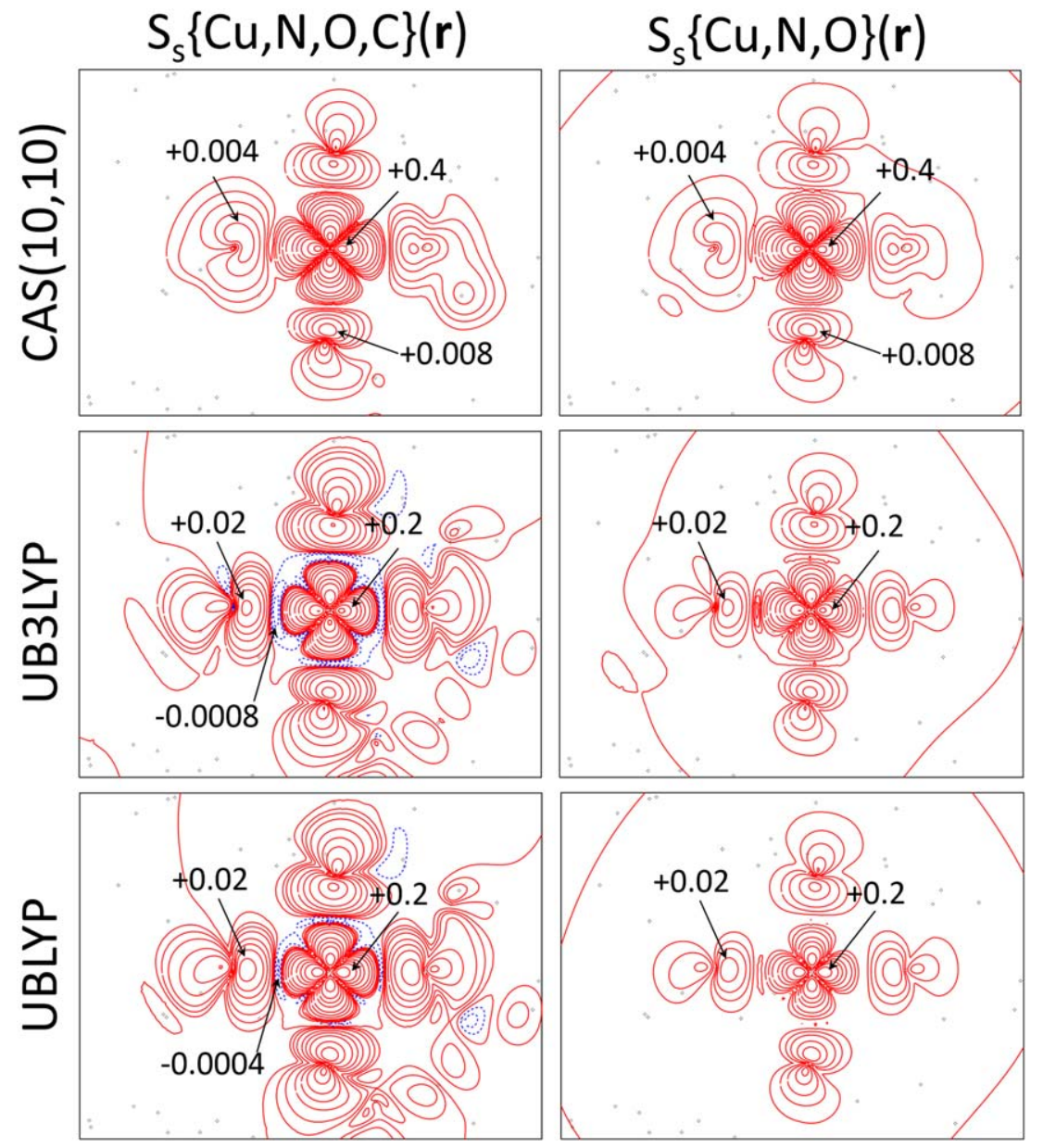

Figure 5. CAS $(10,10)$, UB3LYP, and UBLYP contour plots of the SF reconstructed partial SD in the least-square plane of the $\mathrm{Cu}-\mathrm{O}$ and of the three shorter $\mathrm{Cu}-\mathrm{N}$ bonds. $\{\mathrm{Cu}, \mathrm{N}, \mathrm{O}, \mathrm{C}\}$ (left) denotes the atoms subset containing the two $\mathrm{Cu}$, all $\mathrm{N}$ atoms, the two $\mathrm{O}$ and all the $\mathrm{C}$, while in the $\{\mathrm{Cu}, \mathrm{N}, \mathrm{O}\}$ subset (right) the contribution from the $\mathrm{C}$ atoms has been removed. Color codes, isovalue contours, and plane orientation are the same as in Figure 3. [Color figure can be viewed at wileyonlinelibrary.com]

different. DFT calculations display a positive, spherical shaped SD centered on the $\mathrm{Cu}$ nucleus, surrounded by a negative SD region. The latter is responsible for the presence of the negative $\mathrm{SD}$ region between the $\mathrm{Cu}^{\prime \prime}$ cation and the ligands. On the contrary, the $\operatorname{CAS}(10,10)$ method finds again a $d_{x^{2}-y^{2}}$ angular shape of the relaxation SD, but of opposite sign and lower magnitude relative to the magnetic term. This negative SD relaxation region completely overlaps with that due to the magnetic component, leading just to a decrease of the total SD but not to the onset of a SD negative zone as for the DFT. Moreover, a clearly positive " $\pi$-like" SD distribution is present on the azido N5 nitrogen, facing the $d_{x^{2}-y^{2}}$ SD negative region around the $C u^{\prime l}$ cation.

The SD contours evaluated in the azido least-squares plane are reported in Figures S3 of the Supporting Information. The DFT functionals predict a spin polarization mechanism within the azido moiety, with negative SD regions surrounding the central N4 nucleus. These features of the SD distribution closely resemble those previously found in the EO system. ${ }^{[1]}$ In particular, the EE N3 atom behaves like the EO terminal nitrogen N3, which suggests that the $\mathrm{Cu}$ and N3 atoms hardly share any significant spin information in the EE complex. The main difference concerns the atoms N5 (EE) and N1 (EO), because of their different coordination geometries: in the EO system, the bridging $\mathrm{N} 1$ atom coordinates two $\mathrm{Cu}^{\prime \prime}$ cations and shows a SD distribution oriented along the bonds with the metallic ions, while in the EE complex the N5 atom only coordinates one $\mathrm{Cu}^{\text {Il }}$ cation and has therefore a SD distribution aligned along only this unique $\mathrm{Cu}-\mathrm{N} 5$ bond. $\operatorname{CAS}(10,10)$ calculation does not find any negative SD region around the N4 atom and predicts a spin delocalization mechanism across the azido moiety, in contrast to the DFT results. As for the other investigated least-square plane, the tendency to exaggerate the delocalization of the unpaired electrons on the ligands by the used exchange-correlation functionals is evident.

The Laplacian SD $\nabla^{2} s$ maps for both discussed planes are shown in Figures S4-S5 in the Supporting Information.

\section{Dissecting atomic group contributions: source function partial reconstructions of the spin densities}

Source Function SD partial reconstructions enable us to have an easy to grasp picture on the cause-effect relationship between the basins SD sources and the resulting SD or SD components. Figure 5 shows the reconstruction for the group 

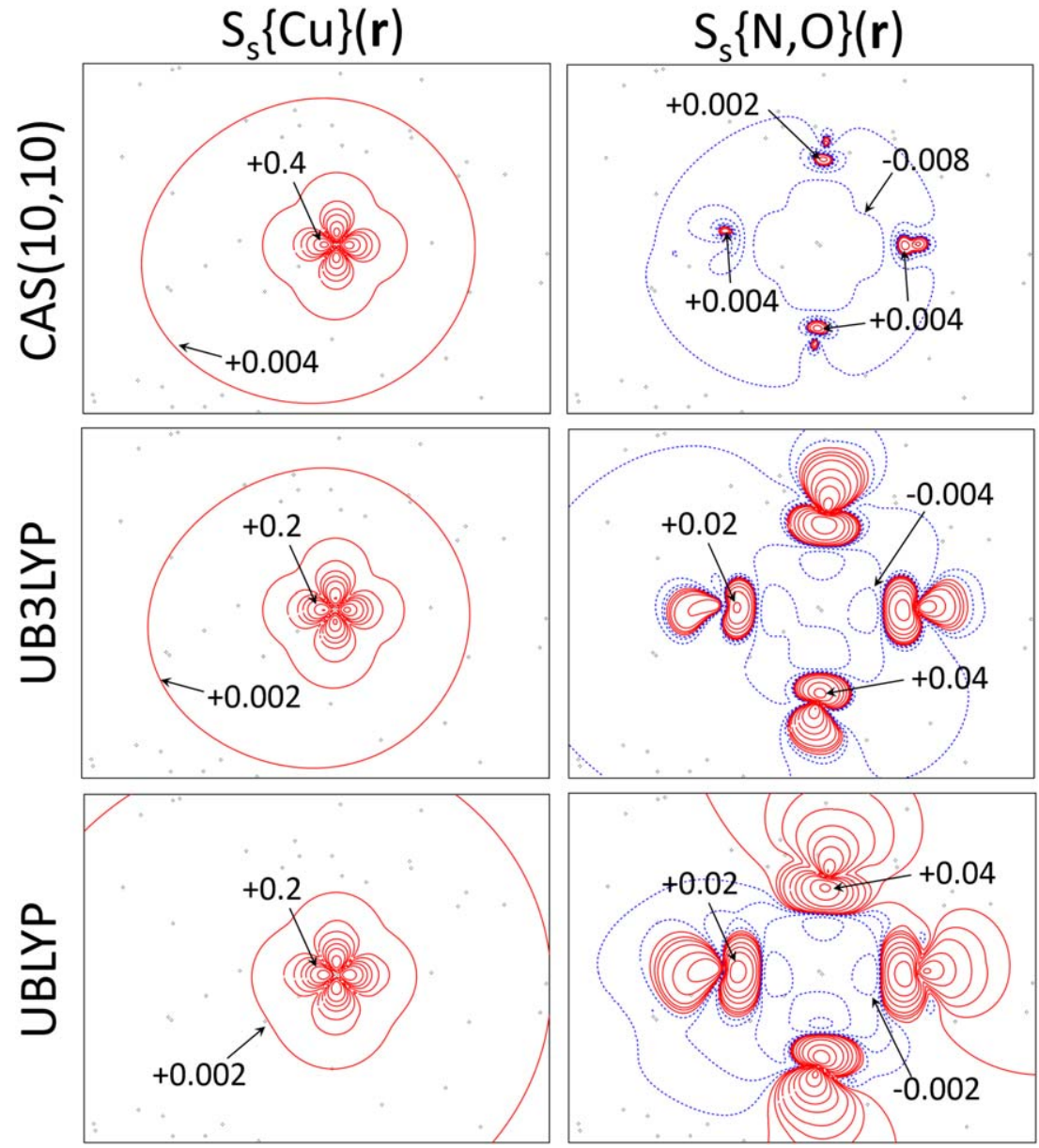

Figure 6. CAS $(10,10)$, UB3LYP, and UBLYP contour plots of the SF reconstructed partial SDs in the least-square plane of the Cu-O and of the three shorter $\mathrm{Cu}-\mathrm{N}$ bonds. $\{\mathrm{Cu}\}$ (left) denotes the subset containing the two $\mathrm{Cu}$ atoms, while $\{\mathrm{N}, \mathrm{O}\}$ subset (right panels) includes the $\mathrm{N}$ and $\mathrm{O}$ atoms only. Color codes, isovalue contours, and plane orientation are the same as in Figure 3. [Color figure can be viewed at wileyonlinelibrary.com]

subsets $\{\mathrm{Cu}, \mathrm{N}, \mathrm{O}, \mathrm{C}\}$ and $\{\mathrm{Cu}, \mathrm{N}, \mathrm{O}\}$, where the condensed notations $\{C \mathrm{C}\},\{\mathrm{N}\},\{\mathrm{O}\}$, and $\{\mathrm{C}\}$ indicate the subsets including the two $\mathrm{Cu}$ atoms, the $\mathrm{N}$ atoms (both azido and $\mathrm{L}$ ligand $\mathrm{N}$ atoms), the two $\mathrm{O}$ atoms and all the $\mathrm{C}$ atoms of the system, respectively.

When the $\{\mathrm{Cu}, \mathrm{N}, \mathrm{O}, \mathrm{C}\}$ subset is used, the primitive SD (directly obtained from the wavefunction and reported in Fig. 4) and the reconstructed one (Fig. 5, first row) are almost indistinguishable, except for small differences on the borders due to numerical errors. This evidence is true for all the used methods, in both considered planes, indicating that the chosen atomic subset includes all the significant atoms for SD reconstruction and that the performed reconstructions are numerically trustable. When the contributions coming from the $\mathrm{C}$ atoms are removed, a few remarkable differences with respect to the primitive SD appear. In the square-pyramidal base plane, the DFT $S_{s}\{C u, N, O\}$ partial reconstructions show the most important changes. Indeed, the negative SD region located between the outermost parts of the $\mathrm{Cu}$ basin and the ligand heteroatoms disappears, due to the contraction of the negative SD region in the relaxation term and of the magnetic SD positive regions around the $\mathrm{Cu}^{\prime \prime}$ cation. The magnetic and relaxation SF partial reconstruction maps are shown in Figures
S6-S8 of the Supporting Information. CAS $(10,10)$ SD reconstruction is less affected by the removal of the $C$ atoms sources, even if a limited contraction of the positive region around the $\mathrm{Cu}$ nucleus is still present. A similar behavior is found in the $\mathrm{N}_{3}^{-}$ plane, where the negative region located on the central $\mathrm{N}$ is reduced when the contribution of the $\mathrm{C}$ atoms is not taken into account (see Supporting Information Figures S9-S11).

The source from the $\mathrm{Cu}$ atoms $\left(S_{s}\{C u\}\right)$ in the squarepyramidal base plane (Fig. 6) closely resembles that found in the EO complex. ${ }^{[1]}$ It retrieves the $d_{x^{2}-y^{2}}$ shape of the primitive SD and it is dominated by its magnetic contribution (Figures S6-S8 in the Supporting Information).

The $\mathrm{Cu}+\mathrm{Cu}^{\prime}$ SD source obtained from the $\operatorname{CAS}(10,10)$ model is higher in magnitude, close to the $\mathrm{Cu}$ nuclei, with respect to the DFT, for both magnetic and relaxation contributions (Figures S7-S8 in the Supporting Information). The result complies with the lower SD delocalization of the CAS wavefunction. In this case, UBLYP $\{C \mathrm{C}\}$ source results more contracted than for UB3LYP. In addition, the source contributions from the four coordinating atoms belonging to the pyramid square base (N5 from the azido moiety and N1, N2, and 01 from the L ligand) are qualitatively similar to those found for the EO complex. ${ }^{[11]}$ The CAS $(10,10)\{\mathrm{N}, \mathrm{O}\}$ subset contribution (Fig. 6) shows highly 


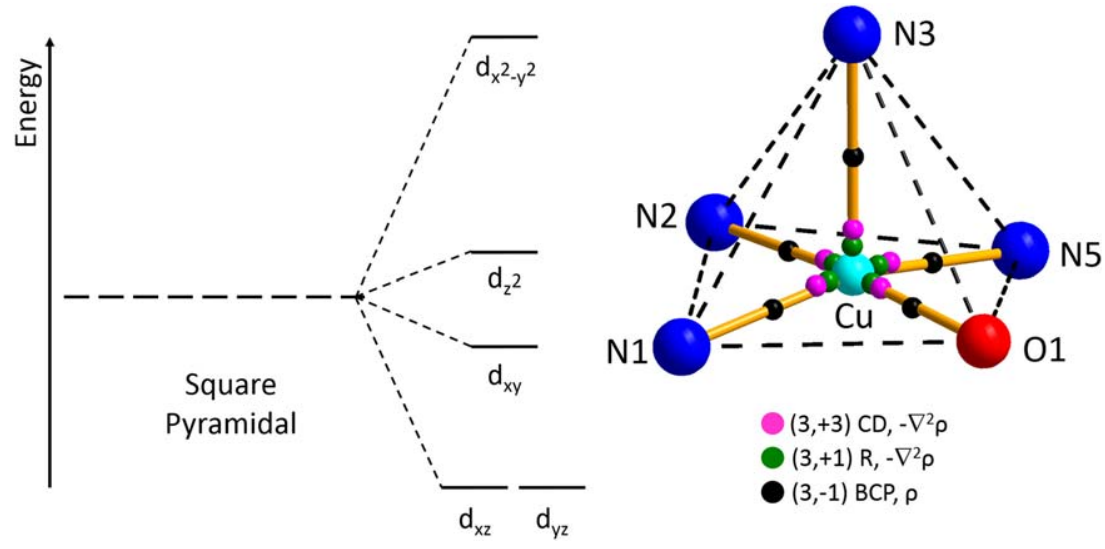

Figure 7. $\mathrm{Cu}^{\prime \prime} 3 d$ electron asphericity for the end-end azido complex. Orbital energy order for a metal atom in a square-pyramidal arrangement of ligands is reported on the left. The locations of the unique critical points of $-\nabla^{2} \rho$ and $\rho$ reported in Table 5 around the Cull cation are shown on the right. [Color figure can be viewed at wileyonlinelibrary.com]

contracted positive regions around the nuclei, surrounded by a diffuse negative region, while DFT functionals maps show a more expanded positive region due to the exaggeration of $\alpha$ spin electron delocalization brought in by DFT methods, as previously discussed. In particular, the UBLYP DFT functional shows more expanded regions of SD sources than the UB3LYP model, which predicts more contracted sources, both positive and negative. These contrasting behaviors for the SF reconstructed SD by the $\mathrm{Cu}$ and its linked atoms subsets, almost cancel out when summed up, thus leading to very close $\{C \mathrm{C}, \mathrm{N}, \mathrm{O}\} \mathrm{SD}$ reconstructed maps for the two functionals here adopted. It is worth noting that the large regions of positive SD around N5 and the other ligand atoms, present in the total SD maps (Fig. 4), have different origins. In the case of CAS $(10,10)$ wavefunction, they are essentially due to the source from the $\mathrm{Cu}$ atom, while for the DFT functionals they mainly originate from their own sources.

In the azido $\mathrm{N}_{3}^{-}$plane, $\operatorname{CAS}(10,10)$, and DFTs $\left\{\mathrm{N}_{\mathrm{Az}}\right\}$, subset sources show the same differences described above: (i) the positive SD region located on N3 and N5 is highly contracted in the CAS $(10,10)$ case; (ii) UBLYP positive SD regions are more expanded than the UB3LYP ones (see Supporting Information Figures S12-S14).

The contour plots of the SF reconstruction in the azido $\mathrm{N}_{3}^{-}$ least-square plane due to the $\mathrm{N}$ atoms belonging to the bridging moiety are similar to those found for the EO complex (see Supporting Information Figure S12-S14). ${ }^{[1]}$ N5 (EE) behaves similarly to N1 (EO). It produces strong positive SD contributions around its nucleus (again more contracted for the CAS method than for the DFT functionals), directed toward the $\mathrm{Cu}^{\prime \prime}$ cation, while yielding a diffuse $\beta$ effect spread in the whole space. The N3 (EE) atom behaves instead as the terminal N3 (EO) atom, contributing with an $\alpha$ effect everywhere. N4 (EE) contrasts such action of the N3 atom, yielding a $\beta$ effect also everywhere. Except for the N4 atom, the magnetic terms are the prevailing contributions in the SF SD reconstruction. Contour plots of these contributions, together with other not mentioned here, are shown in the Figure S6-S14 of the Supporting Information.
$\mathrm{Cu}^{\text {II }} 3 d$ electrons asphericity: electron density Laplacian and electron spin density signatures

In the EE system, the Cu" cation is fivefold coordinated in an almost square-pyramidal arrangement of ligands. According to the crystal field theory, for a $d^{9}$ configuration the $d_{x^{2}-y^{2}}$ is the magnetic singly occupied orbital, as in the EO system. This orbital is directed toward the apexes of the pyramid base, in correspondence with the three external L ligand atoms (N1, $\mathrm{N} 2$, and $\mathrm{O} 1$ ) and with the N5 atom of the azido moiety. The apical ligand $\mathrm{N} 3$, linked to the $\mathrm{Cu}$ by the longer Cu-azide bond, is directed along $z$ (see Fig. 1) and interacts with the almost doubly filled $\mathrm{Cu} \mathrm{d}_{\mathrm{z}^{2}}$ orbital, rationalizing why this bond is definitely longer than $\mathrm{Cu}-\mathrm{N} 5$. Based on this simple model picture, one anticipates that the Laplacian of the ED will exhibit a characteristic departure from spherical symmetry around the metal cation. ${ }^{[32-35]}$ Analogously to the EO system, ${ }^{[11]}$ the topological analysis of $-\nabla^{2} \rho$ in the Valence Shell Charge Concentration (VSCC ${ }^{19}$ ) and Valence Shell Charge Depletion $\left(\operatorname{VSCD}{ }^{19}\right)$ regions of the $\mathrm{Cu}$ atoms finds $(3,+3)-\nabla^{2} \rho$ minima, that is, charge depletions (CDs), all almost lying along the $\mathrm{Cu}$-ligand internuclear axes and $(3,-3)-\nabla^{2} \rho$ maxima, that is, charge concentrations (CCs), lying in between these CDs. CDs locations are shown in Figure 7.

Due to the different nature of the $\mathrm{Cu}^{\text {Il }}$ orbital involved in the apical bond, the CDs along the Cu-ligands bonds of the base of the pyramid are expected to be definitely more evident than along the apical bond. Likewise, using the complementary view offered by the SD distribution, large positive SD values are anticipated at the CDs relative to the bonds in the square-pyramidal base and a small, if not negligible, SD value at the CD of the apical bond. Data in Table 5 nicely confirm all these conjectures.

At the $\mathrm{Cu}-\mathrm{N} 3 \mathrm{CD}, \nabla^{2} \rho$ is less positive (13.65 au at the CAS $(10,10)$ level) than at the Cu-N5 CD $(19.10 \mathrm{au})$, meaning that here the ED is less depleted. The SD value at the $\mathrm{Cu}-\mathrm{N} 3 \mathrm{CD}$ is almost zero while for the $\mathrm{Cu}-\mathrm{N} 5 \mathrm{CD}$ point it assumes a very large value $(s=0.363$ au at $\operatorname{CAS}(10,10)$ level, 0.282 and 0.226 au for UB3LYP and UBLYP models). A $(3,+1)\left|-\nabla^{2} \rho\right|$ Ring ( $R$, Table 5) critical point is also found along all $\mathrm{Cu}$-ligand axes and located at a distance from the Cu nucleus very close 
Table 5. Distances from the Cu nucleus, $R_{C_{u}}$ electron density Laplacian, $\nabla^{2} \rho(\boldsymbol{r})$, spin density, $s(\boldsymbol{r})$, and its magnetic and relaxation components, $s_{\text {mag }}(\boldsymbol{r})$ and $s_{\text {relax }}(\boldsymbol{r})$, evaluated at selected critical points (CPs) along the long, $\mathrm{Cu}-\mathrm{N} 3$, and the short, $\mathrm{Cu}-\mathrm{N} 5$, bonds of $\mathrm{Cu}$ " cation with the azido moiety.

\begin{tabular}{|c|c|c|c|c|c|c|}
\hline Bond & $\mathrm{CP}^{[\mathrm{a}]}$ & $R_{\mathrm{Cu}}(\AA)$ & $\nabla^{2} \rho(\boldsymbol{r})$ & $s(\boldsymbol{r})$ & $s_{\text {mag }}(r)$ & $S_{\text {relax }}(\boldsymbol{r})$ \\
\hline \multirow{3}{*}{ Cu-N3 } & $-\nabla^{2} \rho(3,+3), C D$ & $0.45(0.45 / 0.45)$ & $13.65(14.14 / 14.26)$ & $0.000(0.003 / 0.006)$ & $0.000(0.001 / 0.003)$ & $0.000(0.002 /(0.002)$ \\
\hline & $-\nabla^{2} \rho(3,+1), \mathrm{R}$ & $0.28(0.28 / 0.28)$ & $-69.88(-68.73 /-68.06)$ & $0.001(0.013 / 0.022)$ & $0.001(0.003 / 0.013)$ & $0.000(0.011 / 0.009)$ \\
\hline & $\rho, \mathrm{BCP}$ & $1.16(1.18 / 1.19)$ & $0.15(0.14 / 0.14)$ & $0.000(0.000 / 0.000)$ & $0.000(0.000 / 0.000)$ & $0.000(0.000 / 0.000)$ \\
\hline \multirow[t]{3}{*}{ Cu-N5 } & $-\nabla^{2} \rho(3,+3), C D$ & $0.43(0.43 / 0.44)$ & $19.10(17.93 / 17.30)$ & $0.363(0.282 / 0.226)$ & $0.404(0.279 / 0.223)$ & $-0.041(0.004 / 0.002)$ \\
\hline & $-\nabla^{2} \rho(3,+1), \mathrm{R}$ & $0.29(0.29 / 0.29)$ & $-29.48(-40.36 /-46.59)$ & $0.913(0.770 / 0.637)$ & $1.016(0.753 / 0.625)$ & $-0.103(0.016 / 0.012)$ \\
\hline & $\rho, \mathrm{BCP}$ & $0.94(0.97 / 0.98)$ & $0.35(0.26 / 0.24)$ & $0.006(0.001 / 0.001)$ & $0.007(0.003 / 0.001)$ & $-0.001(-0.002 /-0.001)$ \\
\hline
\end{tabular}

[a] CP legend: $(3,+3), C D$ : Charge Depletion; $(3,+1)$, R: Ring; BCP: bond critical point. Data from the CAS $(10,10)$ wavefunction (UB3LYP/UBLYP results in parentheses). If not otherwise stated, all the values are reported in atomic units.

(0.28-0.29 $\AA$ ) to that of the CCs. This Ring CP region is also associated to the $\mathrm{d}_{\mathrm{x}^{2}-\mathrm{y}^{2}}$ orbital for the Cu-ligand bonds of the base of the pyramid, while it is related to the almost doubly filled $\mathrm{Cu}$ $d_{z^{2}}$ orbital for the apical bond. This motivates why the same differences in the ED Laplacian and SD values between the short and long $\mathrm{Cu}-\mathrm{N}_{\text {azide }}$ bonds can be also found looking at the $\nabla^{2} \rho(3,+1)$ critical points. The $\mathrm{Cu}-\mathrm{N} 5$ ring CP shows a $-\nabla^{2} \rho$ value which is more than twice as large in magnitude for the $\mathrm{Cu}-\mathrm{N} 3$ bond and an extremely high SD value $(s=0.913 \mathrm{au}$, $\mathrm{CAS}(10,10)$ result), while that for $\mathrm{Cu}-\mathrm{N} 3$ is negligibly small $(0.001 \mathrm{au}, \mathrm{CAS}(10,10)$ result).

Although not reported in Table 5, corresponding values at the selected $\mathrm{CPs}$ along $\mathrm{Cu}-\mathrm{N} 1, \mathrm{Cu}-\mathrm{N} 2$, and $\mathrm{Cu}-\mathrm{O} 1$ (see Supporting Information Table S3) all resemble those found along $\mathrm{Cu}-\mathrm{N} 5$ bond and not those along $\mathrm{Cu}-\mathrm{N} 3$ bond, as expected.
In all these points around the $\mathrm{Cu}^{\text {II }}$ cations, the magnetic contributions are the dominating term, while the relaxation components have lower significance. The CAS calculation always shows important negative relaxation contribution to the $C D$ and Ring critical points along the bonds of the base of the pyramid, higher in magnitude relative to the DFT results. Although CASSCF and DFT methods qualitatively agree (except for the relaxation SDs), they show large quantitative differences. Also UB3LYP and UBLYP quantitatively disagree within each other, showing not only that exchange-correlation functionals are not accurate enough but also that SD results strongly depend on the exchange-correlation functional adopted.

Table 5 also reports data for the $\mathrm{Cu}-\mathrm{N} 3$ and $\mathrm{Cu}-\mathrm{N} 5$ BCPs. UB3LYP BCP distances from $\mathrm{Cu}$ nucleus and the $\rho$ and $\nabla^{2} \rho$ values at BCPs compare well with those obtained by Deutsch
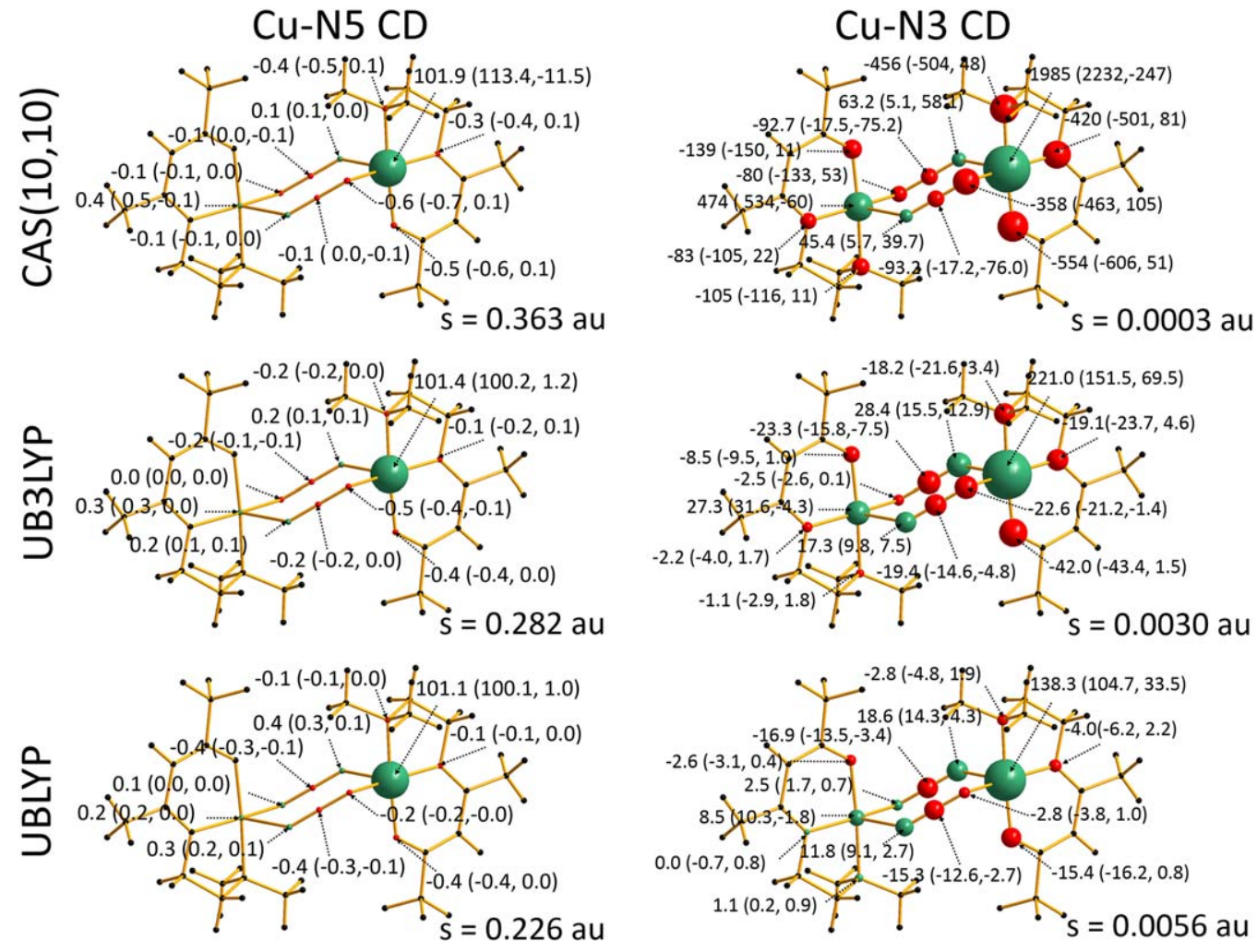

Figure 8. CAS(10,10), UB3LYP, and UBLYP SF percentage contributions to the spin density (magnetic and relaxation components in parentheses) at Cu-N5 and $\mathrm{Cu}-\mathrm{N} 3$ charge depletion (CD) critical points. Green (red) atomic balls denote an $\alpha(\beta)$ effect on the spin density. The radii of the spheres are proportional to the percentage contributions, with $\mathrm{Cu}-\mathrm{N3}$ CD CAS $(10,10)$ spheres volumes reduced by a factor 10 for a better graphical representation. [Color figure can be viewed at wileyonlinelibrary.com] 

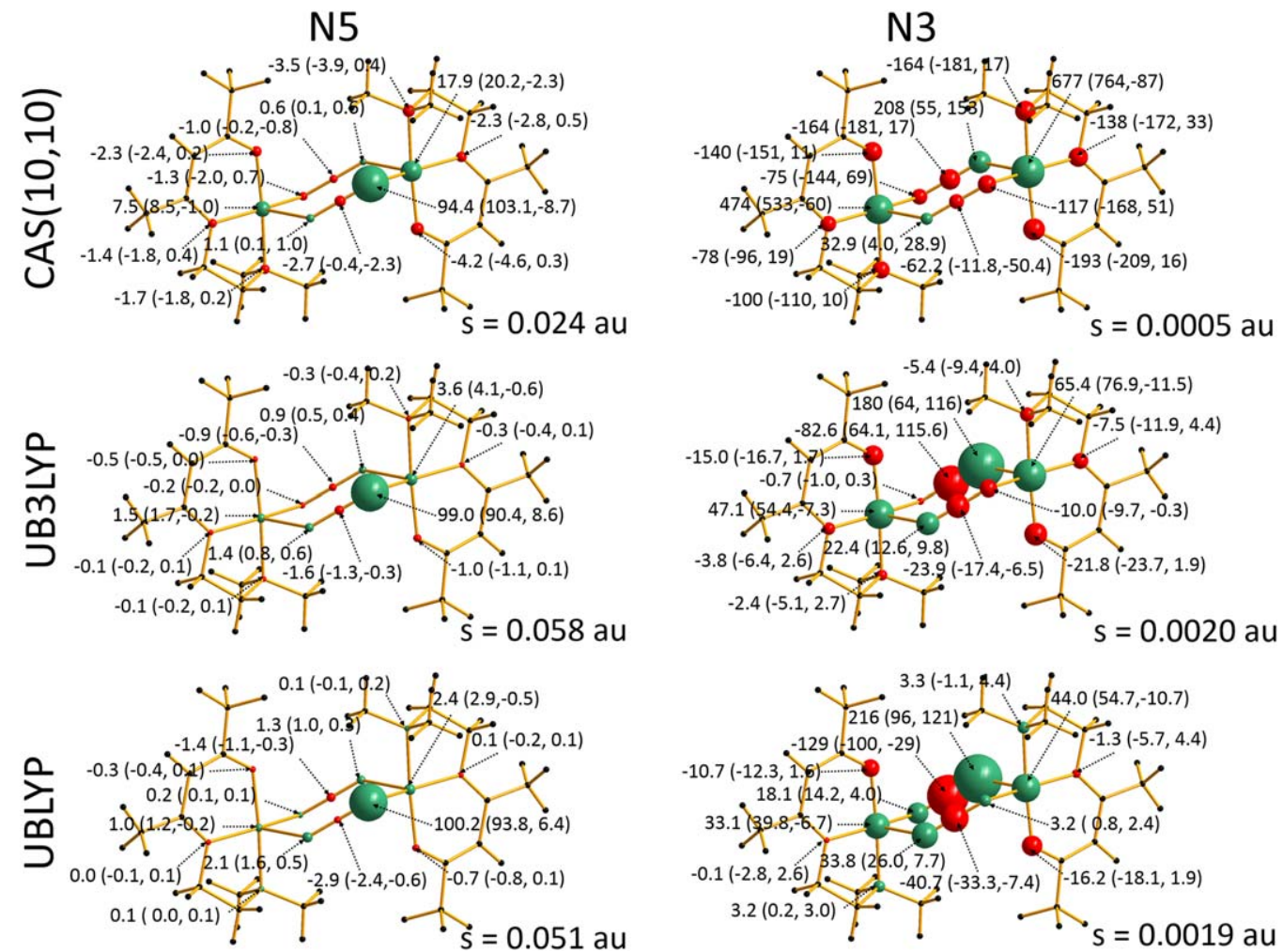

Figure 9. CAS $(10,10)$, UB3LYP, and UBLYP SF percentage contributions to the spin density (magnetic and relaxation components in parentheses) at N5 and N3 nuclei. Green (red) atomic balls denote an $\alpha(\beta)$ effect on the spin density. The radii of the spheres are proportional to the percentage contributions. [Color figure can be viewed at wileyonlinelibrary.com]

et al., using the spin-split multipolar model on X-ray and PND data. ${ }^{[3]}$ SD values from such data refinement are instead more than halved relative to the CAS $(10,10)$ model, which also predicts BCPs locations significantly closer to the metal. As already discussed above and for the EO compound, these BCPs shifts toward the $\mathrm{Cu}$ in the correlated wavefunction may be related to the excessive $\alpha$-spin delocalization and consequent $\mathrm{Cu}$-ligand bond covalency predicted by DFT functionals. Whether this might be also a problem of joint X-ray and PND data refinement, we are unable to judge.

\section{SF percentage analysis at selected reference points}

Spin, magnetic, and relaxation densities in the EE complex and the way these densities are determined from their atomic group sources, in the least-square planes of the $\mathrm{Cu}-\mathrm{O}$ and of the three shorter $\mathrm{Cu}-\mathrm{N}$ bonds of the $\mathrm{N}$-ligands around the $\mathrm{Cu}$ atoms, has been discussed. The $\mathrm{Cu}$ atom $3 d$ electron asphericity and its ED Laplacian and electron SD signatures have been related and properties at a number of peculiar molecular points have been analyzed. Two of these points, along with the N3 and N5 nuclei locations, are now considered as convenient RPs for a conventional SF SD analysis.

Atomic SF percentage contributions for SD reconstruction at CDs critical points (Table 5) along the $\mathrm{Cu}-\mathrm{N} 5$ and $\mathrm{Cu}-\mathrm{N} 3$ bonds are reported in Figure 8. The choice of these two specific RPs was made because we have shown that they have quite different SD properties. Analysis of SF spin density reconstruction at these points could be very useful to get further insight on the mechanism of spin communication between the $\mathrm{Cu}$ atoms and the azido moieties.

$\mathrm{SD}$ at the CD Cu-N5 is dominated by the source of the $\mathrm{Cu}$ atom, whose contribution is close to $100 \%$. For the DFT models, the magnetic component is responsible for almost the totality of the SD (100.2\% and $100.1 \%$ for UB3LYP and UBLYP, respectively), while the relaxation contribution is only about $1 \%$. In the case of $\operatorname{CAS}(10,10)$, the relaxation term is predicted to give a $-11.5 \%$ contribution, far greater than those predicted from DFT. It is also interesting to note that here the relaxation term $(-11.5 \%)$ partially counteracts the magnetic contribution (113.1\%), leading to a total Cu source of $101.9 \%$, comparable to that of the DFT models. In both UB3LYP and UBLYP calculations, the behavior is opposite, as the relaxation source cooperates with the magnetic one, increasing the total $\mathrm{Cu} \mathrm{S}_{\mathrm{s}} \%$. The contributions coming from the other atoms are always lower than $1 \%$, in magnitude. In absolute values, they are however not at all negligible being 1-2 order of magnitude larger than the SD value at the $\mathrm{CD} \mathrm{Cu}-\mathrm{N} 3$. All the adopted methods qualitatively agree in predicting the effect of the ligand atoms. The $\mathrm{L} \mathrm{N1}, \mathrm{N} 2$, and $\mathrm{O} 1$ atoms contrast the $\mathrm{SD}$ at the selected point, which denotes a $\beta$ effect from these basins. The short-bonded N5 and the central N4 atom of the azido moiety also produce $\beta$ effects, while the long-bonded $\mathrm{N} 3$ and the remote $\mathrm{Cu}^{\prime}$ atom cause small $\alpha$ effects. These results are similar to those found for the corresponding SF spin density reconstruction at the $\mathrm{CD}$ along the $\mathrm{Cu}^{\prime}-\mathrm{N} 1$ bond in the EE complex, reported in our previous work, and may be 
similarly interpreted. ${ }^{[1]]}$ The absolute value of SF contributions assesses the local importance of the spin back-delocalization mechanism from the ligands to the paramagnetic center or, in the case of $\mathrm{Cu}$, the spin delocalization between the paramagnetic centers themselves. Then, being the SD positive at the $\mathrm{RP}$, their positive ( $\alpha$ effect) or negative ( $\beta$ effect) sign determines whether they concur or contrast the SD at this CD. Interestingly, the remote $\mathrm{Cu}^{\prime}$ atom, at variance with the N5 atom, concurs to the SD at the $\mathrm{Cu}-\mathrm{N} 5 \mathrm{CD}$, in agreement with the two copper atoms being ferromagnetically coupled through the magnetic density.

The $S_{s} \%$ evaluated at the CD RP along the longer $\mathrm{Cu}-\mathrm{N} 3$ bond reveal a completely different panorama. Here, the contributions are very much delocalized and with large and opposing values, which sum up to an almost zero SD (see Table 5 and Fig. 8). As for the $\mathrm{Cu}-\mathrm{N} 5 \mathrm{CD}$, the adopted methods predict a similar pattern of concurring or opposing sources to the SD reconstruction, with absolute values from the ligand atoms quite close (the only big difference comes from the $\mathrm{Cu}$ atoms) to those found for the short-bond CD. However, percentage sources greatly differ as the values of the SD at the chosen RP are about 2-3 order of magnitude lower than at the Cu-N5 CD and also vary among the methods adopted $(0.0003$ au for CAS $(10,10), 0.0030$ au for UB3LYP, and 0.0056 au for UBLYP). Both magnetic and relaxation terms play an important role in reconstructing the SD at this RP. UB3LYP absolute and percentage sources are in this case found to be generally greater than those of the UBLYP mode, despite the larger SD value at the $\mathrm{Cu}-\mathrm{N} 3 \mathrm{CD}$ point for the latter. It also appears that the communication between the atoms is favored for the former functional.

Figure 9 shows the atomic percentage sources at the N5 and N3 nuclei. SD values at the N5 nucleus (0.024 au CAS(10,10), 0.058 au UB3LYP, and 0.051 au UBLYP) are about 3-4 times smaller than for the bridging $\mathrm{N} 1$ atom in the EO complex $(0.076$ and 0.221 au for $\operatorname{CAS}(6,6)$ and UB3LYP, respectively). ${ }^{[11]}$ This is clearly a sign of less efficient $\alpha$-spin delocalization in the EE relative to the EO complex, in agreement with the much lower coupling constant in the former. In the $\mathrm{EE}$ complex, the role of the N5 atom in the spin coupling mechanism with the other $\mathrm{Cu}^{\prime}$ atom is mediated through $\mathrm{N}^{\prime}$ and $\mathrm{N}^{\prime}{ }^{\prime}$ and is therefore less efficient. The SD values at N3 nucleus (0.0005, 0.0022, and 0.0019 au for CAS $(10,10)$, UB3LYP, and UBLYP, respectively) are similar to the terminal N3 nucleus in the EO complex. The atomic source percentages at the N5 nucleus are qualitatively similar to those found at the N1 nucleus in the EO complex. ${ }^{[11]}$ The main source is due to the atom hosting the RP, which contributes with a percentage ranging between $94 \%$ (CASSCF) and 100\% (UBLYP). The major contribution comes from the magnetic terms, but even relaxation components significantly influence the SD reconstruction. The N5 relaxation source predicted by $\operatorname{CAS}(10,10)$ produces a $\beta$ effect, while both the DFT model sources yield an $\alpha$ effect. The two $\mathrm{Cu}$ atoms and the long-bonded N3 and N3' atoms concur in increasing the SD at the N5 nucleus. As obvious from the coordination geometry, the $S_{s} \%$ from the two $\mathrm{Cu}$ atoms are different. These $\mathrm{Cu}$ sources strongly depend on the level of theory. The CAS method predicts higher \% contributions, but the ratio between the $\mathrm{Cu}$ and $\mathrm{Cu}^{\prime}$ sources remain constantly equal to $\approx 2.4$ for all the methods used. $\operatorname{CAS}(10,10)$ results emphasize the cooperative role of the two $\mathrm{Cu}$ atoms, able to cause together $25.4 \%$ of the SD at the N5 nucleus, compared to a value of only $3-5 \%$ for the two DFT models. Either hybrid or pure DFT thus underestimate spin connection and communication efficiency relative to the CASSCF method. The central atom of the azido moiety (N4) and the other ligand atoms (N1, N2, and O1) all produce negative sources, decreasing the SD value at the N5 nucleus RP.

The negligible SD at the N3 nucleus is the result of comparatively large concurring or opposing sources from a large set of atoms of the molecular complex. Both copper atoms and the N3' nucleus add their $\alpha$ effects to that given by N3, while almost all the other atoms yield a contrasting $\beta$ effect. Contrary to the case of SF reconstruction of SD at the N5 nucleus, the different computational methods disagree on the definition of the dominant source. UB3LYP and UBLYP predict that the major contribution to the SD reconstruction is due to the N3 basins itself, while $\operatorname{CAS}(10,10)$ indicates the two $\mathrm{Cu}$ atoms as the most important sources. This different behavior can be once more explained in terms of the limits of the DFT approach. On the one hand, it exaggerates $\alpha$-spin delocalization from the copper to the azido ligands, and on the other hand, it underestimates the capability of the magnetic centers to transmit their spin information on the neighboring nonmagnetic and magnetic centers.

\section{Conclusions}

In this work, we have presented new evidences on the ability of the SF tool to highlight the spin information transmission mechanisms in magnetic systems. The SF allows for decomposing the SD at any point in the space as a sum of atomic contributions within a cause-effect relationship. This peculiarity results in an increased chemical interpretability of the SD distribution, disclosing how both magnetic and non-magnetic centers cooperate or counteract in defining the SD features. From a practical point of view, understanding the peculiar role of an atom or a group of atoms has strong relevance in the field of magnetic material crystal engineering. Unfortunately, the interpretation of the SD is not as simple as for the ED, because usually it is not immediately clear which set of atoms are contributing to the SD in a given molecular or crystal region and through which mechanism.

SF partial reconstruction of the SD enables one to obtain an immediate visualization of the role played by a selected subset of atoms in determining the SD in a molecular plane or in the space. Such reconstructions also ease the choice of proper RPs where to analyze the reconstruction of the SD through the "classical" $S_{s} \%$ patterns. The decomposition of the SF contributions in terms of magnetic and relaxation components provides further chemical insight and neatly discloses the origin of the SD distribution discrepancies when theoretical approaches of increasing quality are used. 
In this work, we have used all these mentioned SF analyses to discuss the SD distribution of an asymmetrical EE azido Cu" dinuclear complex and to detail analogies and differences with the SD of a symmetric EO azido compound. In particular, we have focused on the performance of different quantum mechanical approaches in retrieving accurate $S D$, relative to the SD obtained with $\operatorname{CAS}(10,10)$ model (the electron correlated $a b$ initio approach with the largest attainable active space for our computational resources). We show that $\operatorname{CAS}(6,6)$ level has not achieved a reasonable active space convergence and that expansion to $\operatorname{CAS}(10,10)$ is required. $\operatorname{CAS}(6,6)$ method tends to underestimate $\alpha$-spin delocalization from the paramagnetic centers toward the ligands relative to the $\operatorname{CAS}(10,10)$ model. Instead, comparison of the $\operatorname{CAS}(10,10)$ results with those from UDFT reveals that both hybrid UB3LYP and pure UBLYP functionals exaggerate $\alpha$-spin delocalization toward the ligand atoms. DFT methods also predict large spin polarization regions between the $\mathrm{Cu}$ atoms and the linked ligands atoms. Formation of these regions is likely a consequence of the well known excessive electron delocalization inherent to DFT, favoring an increased covalency of the metal-ligand bonds, relative to CAS. The polarization mechanism, whose sources extend up to the $C$ atoms of the $L$ ligands, is missing in the $\operatorname{CAS}(10,10)$ SD distribution. The decreased or the enhanced $\alpha$-spin delocalization to the ligands complies with a significantly different spin population on the $\mathrm{Cu}$ atom which increases (decreases) for $\operatorname{CAS}(6,6)$ (DFT) methods relative to $\operatorname{CAS}(10,10)$. Concerning DFT approaches, discrepancy with respect to the $\operatorname{CAS}(10,10)$ results is more evident for the pure UBLYP exchangecorrelation functional than for UB3LYP, contrary to what Boguslawski et al. found for the Fe" nitrosyl complex. ${ }^{[13]}$ Experimental PND SPs are intermediate between the $\operatorname{CAS}(10,10)$ and the UB3LYP ones, suggesting that the SD derived this way is reasonably accurate despite the practical and technical limitations of the PND experiment.

The $\mathrm{Cu} 3 d$ electron distribution asphericities of the $\mathrm{EE}$ and of the EO complexes present analogies for the short Cu-ligand bonds, while the apical bond of the EE system behaves differently involving the almost filled $\mathrm{Cu} \mathrm{d}_{\mathrm{z}^{2}}$ orbital, rather than the lobes of the formally singly occupied $d_{x^{2}-y^{2}}$ orbital. Such difference is reflected in the SF percentage patterns at the charge density depletions along the short and long bonds, the former being very close to those of the $\mathrm{Cu}-\mathrm{N}_{\text {azide }}$ bond in the EO system and the latter being completely different. SF percentage analysis highlights the different magnetic pathways between the $\mathrm{Cu}$ atom and the two terminal $\mathrm{N}$ atoms of the azido group, confirming that $\mathrm{Cu}$ and N3 hardly share spin information. These outcomes, together with the long distance between the two paramagnetic centers, justify the low efficacy of the coupling mechanism, which leads to a low coupling constant $J$ for the EE complex.

SF percentage analyses at the charge density depletions along the short and the long $\mathrm{Cu}-\mathrm{N}_{\text {azide }}$ bonds, as well as those at the N3 and N5 nuclei positions, confirm the limits of the DFT approach. As found for the EO system, DFT exaggerates $\alpha$-spin delocalization from the copper to the azido ligands while underestimates the capability of the magnetic centers to transmit their spin information on the neighboring nonmagnetic and magnetic centers, regardless of the hybrid or pure exchangecorrelation nature of the DFT functional.

Keywords: spin density - source function - spin information transmission - spin density accuracy - azido $\mathrm{Cu}$ dinuclear complexes

How to cite this article: G. Macetti, L. Lo Presti, C. Gatti. J. Comput. Chem. 2018, 39, 587-603. DOI: 10.1002/jcc.25150

Additional Supporting Information may be found in the online version of this article.

[1] B. Gillon, C. Sangregorio, A. Caneschi, D. Gatteschi, R. Sessoli, E. Ressouche, Y. Pontillon, Inorg. Chim. Acta 2007, 360, 3802.

[2] M. Deutsch, N. Claiser, S. Pillet, Y. Chumakov, P. Becker, J.-M. Gillet, B. Gillon, C. Lecomte, M. Souhassou, Acta Crystallogr. A 2012, 68, 675.

[3] M. Deutsch, B. Gillon, N. Claiser, J.-M. Gillet, C. Lecomte, M. Souhassou, IUCrJ 2014, 1, 194.

[4] P. Coppens, Phys. Scr. 2015, 90, 058001.

[5] P. Coppens, Angew. Chem. Int. Ed. 2009, 48, 4280.

[6] C. Gatti, P. Macchi, In Modern Charge Density Analysis; C. Gatti, P. Macchi ,Eds.; Springer: Dordrecht, Heidelberg, London, New York, 2012.

[7] P. Coppens, X-ray Charge Density and Chemical Bonding; Oxford University Press: Oxford, UK, 1997.

[8] J. A. Duffy, M. J. Cooper, In Modern Charge Density Analysis; C. Gatti, P. Macchi, Eds. Springer: Dordrecht, Heidelberg, London, New York, 2012; Chapter. 8, pp. 165-180.

[9] B. Gillon, P. Becker, In Modern Charge Density Analysis, C. Gatti, P. Macchi, Eds.; Springer: Dordrecht, Heidelberg, London, New York, 2012; Chapter 8, pp. 277-302.

[10] N. K. Hansen, P. Coppens, Acta Crystallogr. A 1978, 34, 909.

[11] C. Gatti, G. Macetti, L. Lo Presti, Acta Crystallogr. B 2017, 73, 565.

[12] J. Conradie, A. Ghosh, J. Phys. Chem. B 2007, 111, 12621.

[13] K. Boguslawski, C. R. Jacob, M. Reiher, J. Chem. Theory Comput. 2011, 7, 2740.

[14] K. Boguslawski, K. H. Marti, O. Legeza, M. Reiher, J. Chem. Theory Comput. 2012, 8, 1970.

[15] C. Gatti, A. M. Orlando, L. Lo Presti, Chem. Sci. 2015, 6, 3845.

[16] C. Gatti, A. M. Orlando, E. Monza, L. Lo Presti, In Applications of Topological Methods in Molecular Chemistry; R. Chauvin, C. Lepetit, B. Silvi, E. Alikhani, Eds.; Springer International Publishing: Switzerland, 2016; Vol. 22, Chapter 5, pp. 101-129.

[17] R. F. W. Bader, C. Gatti, Chem. Phys. Lett. 1998, 287, 233.

[18] C. Gatti, Struct. Bond. 2012, 147, 193.

[19] R. F. W. Bader, Atoms in Molecules: A Quantum Theory; Oxford University Press: Oxford, UK, 1990.

[20] M. Aebersold, B. Gillon, O. Plantevin, L. Pardi, O. Kahn, P. Bergerat, I. von Seggern, F. Tuczek, L. Öhrström, A. Grand, E. Lelièvre-Berna, J. Am. Chem. Soc. 1998, 120, 5238.

[21] C. Aronica, E. Jeanneau, H. El Moll, D. Luneau, B. Gillon, A. Goujon, A. Cousson, M. A. Carvajal, V. Robert, Chem. Eur. J. 2007, 13, 3666.

[22] M. J. Frisch, G. W. Trucks, H. B. Schlegel, G. E. Scuseria, M. A. Robb, J. R. Cheeseman, G. Scalmani, V. Barone, G. A. Petersson, H. Nakatsuji, X. Li, M. Caricato, A. V. Marenich, J. Bloino, B. G. Janesko, R. Gomperts, B. Mennucci, H. P. Hratchian, J. V. Ortiz, A. F. Izmaylov, J. L. Sonnenberg, D. Williams-Young, F. Ding, F. Lipparini, F. Egidi, J. Goings, B. Peng, A. Petrone, T. Henderson, D. Ranasinghe, V. G. Zakrzewski, J. Gao, N. Rega, G. Zheng, W. Liang, M. Hada, M. Ehara, K. Toyota, R. Fukuda, J. Hasegawa, M. Ishida, T. Nakajima, Y. Honda, O. Kitao, H. Nakai, T. Vreven, K. Throssell, J. A. Montgomery, Jr., J. E. Peralta, F. Ogliaro, M. J. Bearpark, J. J. Heyd, E. N. Brothers, K. N. Kudin, V. N. Staroverov, T. A. Keith, R. Kobayashi, J. Normand, K. Raghavachari, A. P. Rendell, J. C. Burant, S. S. lyengar, J. Tomasi, M. Cossi, J. M. Millam, M. Klene, C. Adamo, R. Cammi, J. W. Ochterski, R. L. Martin, K. Morokuma, O. Farkas, J. B. Foresman, D. J. Fox, Gaussian 16, Revision A.03; Gaussian, Inc.: Wallingford, CT, 2016. 
[23] M. F. Peintinger, D. V. Oliveira, T. Bredow, J Comput. Chem. 2013, 34, 451.

[24] F. W. Biegler-König, R. F. W. Bader, T.-H. Tang, J. Comput. Chem. 1982, 3, 317. AIMPAC download page: http://www.chemistry.mcmaster.ca/ aimpac/imagemap/imagemap.htm

[25] H. Putz, K. Brandenburg, Diamond - Crystal and Molecular Structure, Visualization; Crystal Impact: Bonn, Germany, 1997-2012. Available at: http://www.crystalimpact.com/diamond

[26] K. Momma, F. Izumi, J. Appl. Crystallogr. 2008, 41, 653.

[27] U. von Barth, L. Hedin, J. Phys. C 1972, 5, 1629.

[28] F. Neese, Comput. Mol. Sci. 2012, 2, 73.

[29] P. Mori-Sánchez, A. J. Cohen, Y. Weitao, Phys. Rev. Lett. 2008, 100, 146401.

[30] F. Neese, Coord. Chem. Rev. 2009, 253, 526.
[31] J. P. Malrieu, R. Caballol, C. J. Calzado, C. de Graaf, N. Guihéry, Chem. Rev. 2014, 114, 429.

[32] R. Bianchi, C. Gatti, V. Adovasio, M. Nardelli, Acta Crystallogr. B 1996, $52,471$.

[33] P. Macchi, A. Sironi, Coord. Chem. Rev. 2003, 238-239, 383.

[34] C. Gatti, Z. Kristallogr. 2005, 220, 399.

[35] L. Lo Presti, R. Destro, J. Chem. Phys. 2008, 128, 044710.

Received: 5 August 2017

Revised: 10 November 2017

Accepted: 8 December 2017

Published online on 5 January 2018 